\title{
Properties of the Liquid-Vapor Interface of Acetone- Methanol Mixtures, as Seen from Computer Simulation and ITIM Surface Analysis
}

Abdenacer Idrissi ${ }^{1}$, György Hantal ${ }^{2,3}$, and Pál Jedlovszky, ${ }^{2,4,5}$,

${ }^{1}$ Laboratoire de Spectrochimie Infrarouge et Raman (UMR CNRS A8516), Université Lille 1, Science et Technologies, 59655 Villeneuve d'Ascq Cedex, France

${ }^{2}$ EKF Department of Chemistry, Leányka utca 6, H-3300 Eger, Hungary

${ }^{3}$ University of Vienna, Institut für Computergestützte Biologische Chemie, Währinger Strasse 17, A-1090 Vienna, Austria

${ }^{4}$ Laboratory of Interfaces and Nanosize Systems, Institute of Chemistry, Eötvös Loránd University, Pázmány P. Stny 1/A, H-1117 Budapest, Hungary

${ }^{5}$ MTA-BME Research Group of Technical Analytical Chemistry, Szt. Gellért tér 4, H-1111 Budapest, Hungary

Running title: Liquid-Vapor Interface of Acetone-Methanol Mixtures

*Electronic mail: pali@chem.elte.hu 


\section{Graphical and textual abstract for the contents page:}

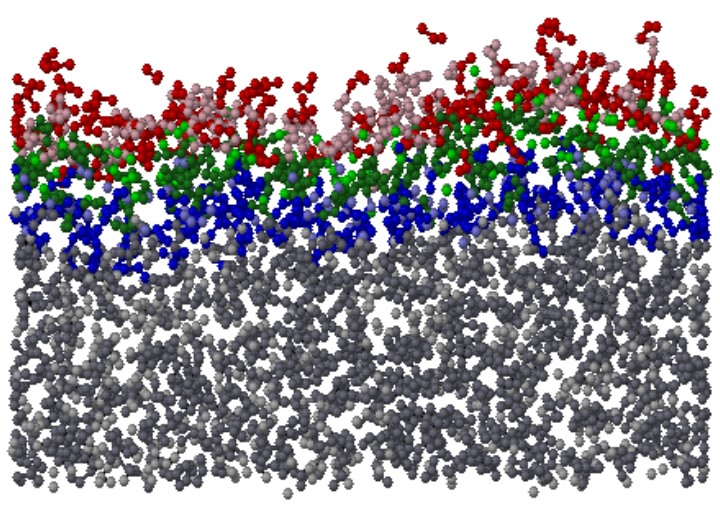

Intrinsic surface of acetone-methanol mixtures is studied by computer simulation and ITIM analysis 


\section{Abstract}

Molecular dynamics simulations of the liquid-vapor interface of acetone-methanol mixtures of different compositions, including the two neat systems have been performed on the canonical $(N, V, T)$ ensemble at $293 \mathrm{~K}$. The intrinsic liquid surface has been determined in terms of the Identification of the Truly Interfacial Molecules (ITIM) method. The results have revealed that the proximity of the interface influences the properties of only the first molecular layer of the liquid phase, while the second layer already turns out to be bulk-like in every respect. The two molecules are distributed uniformly along the macroscopic surface normal axis, as no strong preference for surface adsorption is shown by any of them. However, similarly to the bulk liquid phase, both molecules exhibit a marked tendency for self-association within the surface layer. Surface orientations are found to be composition independent; all the preferred orientations of both molecules correspond to the same alignment of the molecular dipole vector, which is nearly parallel with the macroscopic surface plane, declining only $10-20^{\circ}$ from it towards the vapor phase. Surface properties are thus primarily governed by dipolar interactions, whereas hydrogen bonding within the surface layer, which decreases steadily with increasing acetone mole fraction, plays only a minor role in this respect. 


\section{Introduction}

Acetone is one of the most widely used polar solvents both in preparative chemistry and in the chemical industry. Its solvation properties (e.g., dielectric constant, density, etc.) can be fine tuned by mixing it with other solvents in different proportions. When protic cosolvent (e.g., water, alcohols, etc.) is used the acetone molecules can also participate in the hydrogen bonding network of the co-solvent molecules as $\mathrm{H}$-acceptor. This ability of the acetone molecules is a key factor in determining both the structural and the dynamical properties of these mixtures. ${ }^{1}$

The solvation properties of acetone-alcohol mixtures are strongly related to the ratio of the apolar, strongly polar but aprotic, and H-donor groups present in the system, which ultimately depends on the molar ratio of the two components. Acetone-methanol mixtures are the simplest examples of such solvent mixtures, as they contain all the above three elements of organic solvents in their simplest possible form, i.e., the apolar $\mathrm{CH}_{3}$ groups as well as the polar but aprotic $\mathrm{C}=\mathrm{O}$ groups of the acetone, and $\mathrm{H}$-donor $\mathrm{OH}$ groups of the methanol molecules. Since exactly these kinds of groups play important roles also in bioactive molecules, analysis of the local structure of acetone-methanol mixtures may also provide some insight in the processes laying behind biologically relevant interactions.

Because of the markedly different chemical character of the three constituent groups, acetone-methanol mixtures are highly non-ideal, showing maximum pressure azeotropic behavior. ${ }^{2,3}$ The non-ideal mixing of these components is a macroscopic reflection of the fact that both molecules show self-association behavior in their mixtures, which leads to the occurrence of microheterogeneities in these systems. ${ }^{4-6}$ In a recent study we have shown that the self-association of the like molecules in these systems originates from the fact that the mixing of acetone with methanol is an energetically unfavorable process, and the full miscibility of the two molecules is primarily governed by the entropy gain of ideal mixing. ${ }^{7}$

In contrast to their bulk phase properties, little is known, however, about the surface of acetone-methanol mixtures. Clearly, in the presence of an apolar interface the interplay of the three main structural elements of these mixtures becomes different from that in the bulk liquid phase, which may even alter the self-association behavior of the like molecules at such interfaces. This fact can be of great importance in the field of heterogeneous, and, in particular, heterogeneous catalytic reactions. Therefore, the detailed investigation of the 
liquid-vapor interface of acetone-methanol mixtures is of great scientific interest both from a purely theoretical and also from a practical point of view.

Computer simulation methods provide an excellent tool to get detailed insight into the molecular level structural and dynamical properties of condensed disordered systems. However, in the simulation of fluid interfaces one has to face the difficulty that such interfaces are corrugated by the presence of capillary waves. In other words, such interfaces are rough on the molecular scale, and hence the determination of the exact location of the interface itself in a computer simulation is not a trivial task at all. The majority of the early computer simulations of interfacial systems simply neglected this problem by defining the interfacial region as a slab parallel with the Gibbs dividing surface in which the density of the components fall between the values characteristic to the two bulk phases. However, it has later been shown that this simple treatment of the interface leads to a systematic error of unknown magnitude of the calculated structural properties ${ }^{8,9}$ and composition ${ }^{10-12}$ of the surface layer, and this error can even propagate into some thermodynamic properties of the two-phase system investigated. ${ }^{13}$

The need of finding the position of the molecularly rough, capillary wave-corrugated, so-called intrinsic interface was already recognized in the very first simulations of fluid interfacial systems. Thus, in their pioneering works Linse ${ }^{14}$ and Benjamin ${ }^{15}$ divided the simulation box into several slabs parallel with the interface normal axis, and determined the position of the interface in each slab separately. Later, Jorge and Cordeiro determined the number of slabs required for convergence to the intrinsic surface. ${ }^{16}$ Pandit et al. determined the two dimensional Voronoi tessellation of the projections of the surface molecules in the plane of the Gibbs dividing surface, and approximated the intrinsic surface by lifting the Voronoi cells into the original position of their central particles along the surface normal axis. ${ }^{17}$ Chacón and Tarazona defined the intrinsic surface in a self-consistent algorithm as the surface of minimum area covering a set of pivot atoms. ${ }^{18,19}$ Chowdhary and Ladanyi used a criterion involving the distance from molecules of the opposite phase to detect the intrinsic liquid-liquid interface. ${ }^{20}$ The extension of this algorithm by introducing a scalable parameter was shown to give results that are consistent with those of other intrinsic surface analyzing methods. ${ }^{21}$ Several methods that are even free from the assumption that the interface is macroscopically flat, and thus able to analyze also the surface of, e.g., proteins or micelles, have also been proposed in the past decade. ${ }^{22-24}$ The great physical relevance of the determination of the intrinsic liquid surface in computer simulation studies has recently been 
demonstrated by explaining the experimentally known but yet unexplained surface tension anomaly of water using intrinsic surface analysis. ${ }^{25,26}$

A few years ago we have developed a method, called Identification of the Truly Interfacial Molecules (ITIM), which detects the intrinsic surface of a liquid phase by identifying the molecules that are right at its boundary with the opposite phase. ${ }^{8}$ Comparing its performance with that of other intrinsic methods, ITIM turned out to be an excellent compromise between accuracy and computational cost. ${ }^{21}$ The additional advantage of the ITIM method is that it readily provides the full set of the interfacial molecules, allowing a straightforward analysis of various structural and dynamical properties of the interface. Further, by disregarding the set of molecules identified as interfacial and repeating the entire algorithm, the full set of molecules constituting the second subsurface layer can also be identified, and this procedure can be continued by identifying the subsequent subsurface layers until the physical relevance of such layers is lost. ${ }^{8}$ The ITIM method has successfully been applied to a number of liquid-liquid $d^{9,13,27,28}$ and liquid-vapor ${ }^{8,10-12,25,26,29-38}$ interfaces, including, among others, the liquid-vapor interface of neat methanol ${ }^{10}$ and acetone, ${ }^{38}$ and also to determine the intrinsic free energy profile of ionic and non-ionic penetrants across such interfaces. $^{39,40}$

In this paper, we present a detailed investigation of the liquid-vapor interface of acetone-methanol mixtures in the entire composition range from neat acetone to neat methanol on the basis of molecular dynamics computer simulations and ITIM analysis, using a potential model combination that is able to excellently reproduce the mixing properties of the two components. ${ }^{7}$ For comparisons, the ITIM analysis is also extended to the second and third subsurface molecular layers. Particular attention is paid to the self-association of the like molecules both within the surface layer and along the surface normal (i.e., adsorption in the surface and various subsurface layers). Further, the surface orientation of the molecules, the dynamics of their exchange between the surface layer and the bulk liquid phase, and the molecular scale roughness of the intrinsic surface are also analyzed in detail.

The paper is organized as follows. In Section 2 details of the molecular dynamics simulations and ITIM analyses performed are given. The obtained results are discussed in detail in Section 3, whereas in Section 4 the main conclusions of this study are summarized. 


\section{Computational details}

\subsection{Molecular dynamics simulations}

Molecular dynamics simulations of the liquid-vapor interface of acetone-methanol mixtures of different compositions have been performed on the canonical $(N, V, T)$ ensemble at the temperature of $293 \mathrm{~K}$. The length of the $X$ edge of the rectangular basic simulation box, being perpendicular to the liquid-vapor interface has been $200 \AA$, whereas the cross section of the basic box in the $Y Z$ plane has been $70 \AA \times 70 \AA$. The basic box has contained the total number of 4000 molecules. Besides the two neat systems, simulated for reference purposes, mixtures of nine different compositions, corresponding to the acetone mole fraction $\left(x_{\mathrm{ac}}\right)$ values of $0.1,0.2,0.3,0.4,0.5,0.6,0.7,0.8$, and 0.9 have been simulated.

Methanol molecules have been described by the Transferable Potential for Phase Equilibria (TraPPE) force field, ${ }^{41}$ whereas for acetone a modified version of the TraPPE potential $^{4}$ has been used. This model combination was shown to reproduce the maximum pressure azeotropic behavior of acetone-methanol mixtures ${ }^{4}$ and provides an excellent reproduction of the thermodynamic changes accompanying the mixing of the two components. $^{7}$ According to the potential models used, the total potential energy of the systems have been calculated as the sum of the pair interaction energies, and the $u_{i j}$ interaction energy of the molecule pair $i$ and $j$ has been assumed to be the sum of the Lennard-Jones and chargecharge Coulomb contributions of all of their interaction sites:

$$
u_{i j}=\sum_{a}^{n_{i}} \sum_{b}^{n_{j}} \frac{1}{4 \pi \epsilon_{0}} \frac{q_{a} q_{b}}{r_{i a, j b}}+4 \varepsilon_{a b}\left[\left(\frac{\sigma_{a b}}{r_{i a, j b}}\right)^{12}-\left(\frac{\sigma_{a b}}{r_{i a, j b}}\right)^{6}\right] .
$$

In this equation, indices $a$ and $b$ run over the $n_{i}$ and $n_{j}$ interaction sites of molecules $i$ and $j$, respectively, $\epsilon_{0}$ is the vacuum permittivity, $q_{a}$ and $q_{b}$ are the fractional charges carried by the respective interaction sites, $r_{i a, j b}$ is the distance between site $a$ of molecule $i$ and site $b$ of molecule $j$, whereas $\varepsilon_{a b}$ and $\sigma_{a b}$ are the Lennard-Jones energy and distance parameters, respectively, of the site pair $a$ and $b$, related to the parameters corresponding to the individual sites through the Lorentz-Berthelot rule: ${ }^{42}$

$$
\varepsilon_{a b}=\sqrt{\varepsilon_{a} \varepsilon_{b}}
$$

and 


$$
\sigma_{a b}=\frac{\sigma_{a}+\sigma_{b}}{2}
$$

The $q, \varepsilon$ and $\sigma$ interaction parameters of the acetone and methanol models used here are summarized in Table 1. All interactions have been truncated to zero beyond the molecular center-based cut-off distance of $15 \AA$. The long range part of the electrostatic interaction has been accounted for by the method of Ewald summation in its Particle Mesh Ewald (PME) implementation. $^{43}$

Both acetone and methanol molecules have been rigid in the simulations, the bond lengths and bond angles of the originally flexible models have been kept fixed at their equilibrium values. Thus, the $\mathrm{CH}_{3}-\mathrm{C}$ and $\mathrm{C}=\mathrm{O}$ bond lengths, and $\mathrm{CH}_{3}-\mathrm{C}=\mathrm{O}$ and $\mathrm{CH}_{3}-\mathrm{C}-\mathrm{CH}_{3}$ bond angles of the planar acetone molecule have been $1.520 \AA, 1.229 \AA, 121.4^{\circ}$, and 117.2 ${ }^{\circ}$, respectively, whereas the $\mathrm{CH}_{3}-\mathrm{O}$ and $\mathrm{O}-\mathrm{H}$ bond lengths and $\mathrm{CH}_{3}-\mathrm{O}-\mathrm{H}$ bond angle of the methanol molecule have been set to $1.430 \AA, 0.945 \AA$, and $108.5^{\circ}$, respectively. The $\mathrm{CH}_{3}$ groups of both molecules have been treated as united atoms. The geometry of the molecules has been kept fixed by means of the LINCS algorithm. ${ }^{44}$

The simulations have been performed using the GROMACS 4.5.5 program package. ${ }^{45}$ The equations of motion have been integrated in time steps of $1 \mathrm{fs}$. The temperature of the system has been controlled by the Nosé-Hoover thermostat. ${ }^{46,47}$ Initial configurations have been generated by randomly placing the required number of molecules to a basic simulation box the $Y$ and $Z$ axes of which were $70 \AA$ and the length of the $X$ axis roughly corresponded to the density of the simulated liquid mixture. ${ }^{48}$ Overlaps of the molecules have been eliminated by a short $\left(1-5 \times 10^{6}\right.$ step long) Monte Carlo run. The systems have then further equilibrated by performing a $1 \mathrm{~ns}$ long molecular dynamics run on the isothermal-isobaric $(N, p, T)$ ensemble at 1 bar in such a way that only the $X$ axis of the basic box has been allowed to change. The interfacial system has then been created by increasing the $X$ axis of the basic box to $200 \AA$. The interfacial system has then further been equilibrated on the canonical $(N, V, T)$ ensemble for 2 ns. Finally, in the 2 ns long production stage, 2000 sample configurations, separated by 1 ps long trajectories from each other, have been dumped for the analyses.

\subsection{ITIM analyses}

To identify the molecules that constitute the surface layer of the liquid acetonemethanol mixtures the ITIM method ${ }^{8}$ has been used. In an ITIM analysis, probe spheres of a given radius are moved along a set of test lines parallel with the interface normal axis, starting 
from the opposite bulk phase. Once the probe sphere touches the first molecule of the liquid phase it is stopped, the touched molecule is marked as being interfacial, and the procedure continues with the next probe sphere along the next test line. Once all the probe spheres are stopped the full set of the interfacial molecules are identified, while the molecularly rough interface itself is approximated by the set of points at which the probe spheres are stopped. ${ }^{8}$ In defining the situation when a molecule is touched by the probe, their atoms have been represented by spheres the diameter of which has been the corresponding Lennard-Jones distance parameter, $\sigma$. A molecule has been considered as being touched when any of its atoms became in contact with the probe sphere. The distance of two neighboring test lines has been $0.5 \AA$ in the $Y Z$ plane, thus, a grid of $140 \times 140$ test lines has been used. The radius of the probe sphere has been set to $2 \AA$, in order to ensure that it is in the order of the size of the molecules. ${ }^{8}$

Having the molecules of the interfacial layer identified, the entire procedure has been repeated twice more, disregarding the molecules identified in the previous steps. This way, the molecules constituting the second and third molecular layers beneath the liquid surface have also been identified, and the properties of these subsurface layers have also been analyzed. Finally, all quantities calculated have not only been averaged over the 2000 sample configurations, but also over the two liquid-vapor interfaces present in the basic simulation box. An equilibrium snapshot of the equimolar system simulated, indicating also the molecules forming the first three layers beneath the liquid surface is shown in Figure 1.

\section{Results and discussion}

\subsection{Density profiles}

The number density profiles of the acetone and methanol molecules along the interface normal axis, $\underline{X}$, of selected systems are shown in Figure 2. (The position of the acetone and methanol molecules have been represented by those of their central $\mathrm{C}$ atom and $\mathrm{CH}_{3}$ group, respectively, throughout the paper.) As is seen, all these profiles change smoothly from the bulk liquid phase value to zero, suggesting that none of the two components are adsorbed at the liquid surface. To further investigate this point we have plotted the composition (in terms of acetone mole percentage) of the first three molecular layers beneath the surface as a function of the bulk liquid phase composition in Figure 3. As is seen, the acetone content of the first molecular layer exceeds slightly, by $2-4 \%$ that of the bulk liquid phase, but the 
composition of the second and third subsurface molecular layers do not differ noticeably from that of the bulk phase. In other words, acetone has only a very weak tendency for surface adsorption in these mixtures, and this adsorption never goes beyond the first molecular layer. This behavior is in a marked contrast with that of water-methanol and water-dimethyl sulfoxide (DMSO) mixtures, in which the surface layer was found to be considerably richer in the solute molecule than the bulk liquid phase, ${ }^{10,12}$ and also with that of water-acetonitrile and water-HCN mixtures, in which such a surface adsorption was found to involve more than one molecular layers beneath the surface. ${ }^{11,37}$

Figure 2 also shows the mass density profiles of the entire systems simulated as well as of their first layer beneath the surface. As is seen, the mass density profile of all the systems simulated converges to the same value in the bulk liquid phase, in accordance with the fact that the experimental density of both liquid methanol and acetone is $0.79 \mathrm{~g} / \mathrm{cm}^{3}$ at $293 \mathrm{~K}$ and atmospheric pressure. ${ }^{49}$ It is also seen that the density peak of the first molecular layer gives a large contribution to the $X$ range corresponding to the constant density part of the system in every case. Furthermore, as seen from Figure 4, showing the mass density profile of the first three subsurface molecular layers along with that of the entire system for selected compositions, the density peaks of the second and third molecular layers give nonnegligible contributions to the $X$ range of intermediate densities in every case. These findings stress once more the importance of using intrinsic surface analysis in simulations of fluid interfaces, as they provide an estimate of the large fraction of molecules that are misidentified either as being or as not being at the liquid surface when the interfacial region is simply defined by the $X$ range of intermediate densities.

The mass density profiles of the first three subsurface molecular layers turned out to be of Gaussian shape (see Fig. 4) in every case, in accordance with the theoretical argument of Chowdhary and Ladanyi. ${ }^{50}$ Therefore, by fitting a Gaussian function to these profiles one can obtain straightforward measures of both the broadness of the individual layers and the average separation of the subsequent layers. Namely, the peak position, $X_{\mathfrak{c}}$, and width parameter, $\delta$, of the fitted Gaussian function can characterize the average location of the corresponding layer along the macroscopic interface normal axis, $X$, and its width, respectively. Further, the difference of the $X_{\mathrm{c}}$ values of two subsequent molecular layers, $\Delta X_{\mathrm{c}}$, characterizes the average separation of these layers.

The $X_{\mathrm{c}}$ and $\delta$ values corresponding to the first three subsequent molecular layers of all the systems simulated are collected in Table 2. Further, the dependence of the $\delta$ and $\Delta X_{\mathrm{c}}$ 
parameters on the bulk liquid phase composition is plotted in Figure 5. As is seen, the first molecular layer is always noticeably, by about $2-4 \%$ broader than the second, and by about 4-8\% broader than the third subsurface layer. This finding indicates that the effect of the proximity of the vapor phase on the packing of the molecules does not vanish beyond the first molecular layer. Further, the separation of the first and second layers is consistently somewhat (i.e., by 0.5-2\%) larger than that of the second and third layers, and this difference increases with increasing acetone concentration.

It is also seen that the $\delta$ values corresponding to all the three layers considered show a marked maximum in the bulk phase acetone mole fraction range of $70-90 \%$, indicating that the surface of these systems is noticeably more diffuse than that of the systems of other compositions. Interestingly, this composition range coincides with that where the energy change accompanying the mixing of the two components is maximum. ${ }^{7}$ This relatively high energy cost of the mixing could thus be responsible for the less compact packing of the molecules at the liquid surface of these systems.

Figure 6 compares the number density profiles of the methanol and acetone molecules in the first layer of selected systems. For a better comparison, the two profiles are always scaled to each other. As is seen, the density peak of the methanol molecules is shifted somewhat, i.e., by $1-2 \AA$ towards the vapor phase with respect to that of the acetone molecules at every composition. In other words, within the surface layer the methanol molecules are located, on average, closer to the vapor phase than the acetone molecules. This fact might be related to possible hydrogen bonding patterns between the unlike molecules. This point is addressed in more detail in sub-section 3.5. of this paper.

\subsection{Surface roughness}

Since the ITIM analysis provides a large set of points that can be used as an estimate of the geometric covering surface of the liquid phase, ${ }^{8}$ the use of the ITIM method allows one to characterize also the molecular scale roughness of the liquid surface. However, even if the intrinsic liquid surface is fully known, the quantification of its roughness is far from being a trivial task. Clearly, the characterization of the roughness of any wavy surface requires the use of at least two independent parameters, i.e., a frequency-like and an amplitude-like one. We proposed to determine such a parameter pair in the following way. ${ }^{29}$ The average normal distance, $\bar{d}$, i.e., distance along the macroscopic surface normal axis, $X$ ) of two surface points that are at the lateral distance (i.e., distance within the macroscopic plane of the surface, $Y Z$ ) $l$ 
from each other exhibits a saturation curve as a function of $l$. More specifically, at lateral distances that are considerably smaller than the typical wavelengths of the capillary waves, $\bar{d}$ increases linearly with $l$, while at lateral distances that are considerably larger than that waves $\bar{d}$ does not depend any more on $l$. Thus, the $\bar{d}(l)$ data can be reasonably well fitted by the function

$$
\bar{d}=\frac{a \xi l}{a+\xi l} .
$$

The two parameters of eq. $4, \xi$ and $a$, characterize the steepness of the $\bar{d}(l)$ curve at small $l$ values, and its saturation value at large $l$ values, respectively. In this respect, $\xi$ can be regarded as the frequency-like, while $a$ as the amplitude-like parameter that describes the roughness of the wavy liquid surface. ${ }^{8,29}$ Recently we have shown that the amplitude-like parameter, $a$, defined in this way, is closely related to the surface tension of the system.

The $\xi$ and $a$ parameters obtained for the first three layers beneath the surface of the systems simulated are collected in Table 2 , whereas the $\bar{d}(l)$ roughness curves corresponding to the surface layer of selected systems are shown in Figure 7. As is seen, neither the roughness parameters $\xi$ and $a$, nor the $\bar{d}(l)$ roughness curves are sensitive to the composition of the system: the $\xi$ and $a$ parameters of any two mixed systems do not differ by more than about $15 \%$ and, correspondingly, the $\bar{d}(l)$ curves of the different systems are also rather close to each other. This finding is related to the similar surface affinity of the two molecules, reflected also in the lack of strong adsorption of any of the two components at the liquid surface (Fig. 3.). A much more pronounced difference is seen, however, between the roughness curves corresponding to the first and subsequent molecular layers in any of these systems. This is illustrated by the inset of Fig. 7, comparing the $\bar{d}(l)$ curves of the first three layers of the equimolar system. As is seen from Table 2, the $\xi$ and $a$ values corresponding to the second and third subsurface layers are always 40-50\% smaller than those of the first molecular layer. In other words, the effect of the proximity of the interface on the liquid phase in this respect already vanishes right beyond the first molecular layer.

\subsection{Lateral self-association of the surface molecules}

As it has been shown in a previous sub-section, in spite of their strong self-associating ability in the bulk liquid phase, ${ }^{4-7}$ acetone and methanol molecules do not show microscopic 
separation in their mixtures along the direction perpendicular to the macroscopic plane of the liquid surface. Here we address the question whether they can form a laterally microheterogeneous structure at the liquid surface of their mixtures. The presence of microheterogeneities in binary systems can be easily detected by performing Voronoi analysis. ${ }^{51-53}$ In a two dimensional set of seeds (e.g., molecules at the liquid surface) the Voronoi polygon (VP) of each seed is the locus of points that are closer to this given seed than to any other one. As it was shown by Zaninetti, in the case of homogeneous distribution of the seeds the area (or, in three dimensions, volume) distribution of their VP is of Gaussian shape, whereas in the presence of large density fluctuations (i.e., when the seeds prefer to stay at the vicinity of each other, leaving large empty voids between their aggregates) the VP area distribution exhibits a long, exponentially decaying peak at large area values. ${ }^{54}$ As it was later shown by us, ${ }^{55}$ this property can also be used to detect self-association in binary mixtures in the following way. The VP area distribution calculated by taking only into account the molecules of one of the two components and disregarding the other one, is of Gaussian shape in the lack of self-association (i.e., when both components are uniformly distributed), and exhibits a long, exponentially decaying tail if the molecules of the disregarded component are self-associating (as the self-associates are transformed to large voids by disregarding the molecules of this component). This method has been applied successfully for a set of binary systems both in two ${ }^{10-12,37}$ and in three dimensions. ${ }^{7,55}$

Figure 8 shows the distribution of the VP area, $A$, of the surface molecules, projected to the macroscopic plane of the surface, $Y Z$, by taking both methanol and acetone molecules into account as well as by disregarding one of the two components in the analysis. To emphasize the exponential decay of the large $A$ tail of some distributions, the $P(A)$ curves are shown on a logarithmic scale. As is seen, when both components are taken into account, the resulting distributions are narrow Gaussians, in accordance with the fact that the liquid surface is uniformly covered by the surface molecules. However, when only one component is taken into account in the analysis, and the molecules of the other components are disregarded, the $P(A)$ distributions develop an exponentially decaying tail at large $A$ values (converted to straight lines by the logarithmic scale of Fig. 8), and the decay of this tail becomes slower with increasing mole fraction of the disregarded component (i.e., as the vacancies created by disregarding these molecules get larger).

This finding clearly indicates that, in contrast with the observed lack of microscopic separation along the macroscopic surface normal axis, methanol and acetone show a marked tendency of self-association within the surface layer of the liquid phase of their mixtures. This 
lateral self-association of the like molecules is illustrated in Figure 9, showing equilibrium snapshots of the surface layer of the $20 \%, 50 \%$ and $80 \%$ acetone systems by projecting the molecular centers to the macroscopic plane of the surface, $Y Z$. The extent of such selfassociation can be quantified by calculating the area of the largest circular voids in the $Y Z$ plane when one of the two components is disregarded, and compare this value with the average area occupied by a molecule of the disregarded component at the surface of its neat liquid. In this way, the largest lateral acetone and methanol self-associates are estimated to consist of 6 and 66 molecules in the $10 \%$ acetone, 16 and 16 molecules in the equimolar, and 67 and 7 molecules in the $90 \%$ acetone system, respectively.

\subsection{Dynamics of exchange between the surface layer and the bulk}

The dynamics of exchange of the molecules between the surface layer and the bulk liquid phase can be characterized by the survival probability of the molecules within the surface layer. The survival probability, $L(t)$, is simply the probability that a molecule that is in the surface layer at $t_{0}$ will stay at the surface up to $t_{0}+t$. In order to distinguish between the situations when a molecule permanently leaves the surface, and when it only leaves the surface layer temporarily due to an oscillatory move, departure of a molecule from the surface between $t_{0}$ and $t_{0}+t$ is allowed given that it returns within the time window of $\Delta t$. Considering the characteristic time of molecular vibrations, here we set $\Delta t$ to its conventional value of $2 \mathrm{ps}$. To check that this somewhat arbitrary choice of $\Delta t$ does not affect our results we have repeated the analysis with $\Delta t=1 \mathrm{ps,} \mathrm{which} \mathrm{indeed} \mathrm{left} \mathrm{all} \mathrm{of} \mathrm{our} \mathrm{conclusions}$ unchanged.

Since the departure of a molecule from the liquid surface is a process of first order kinetics, the $L(t)$ survival probability exhibits exponential decay. Thus, by fitting the function $\exp (-t / \tau)$ to the calculated $L(t)$ data the mean residence time of the molecules at the surface, $\tau$, can also be calculated. The $\tau$ values obtained for the acetone and methanol molecules in the different systems simulated are included in Table 2, while the $L(t)$ curves corresponding to the $10 \%, 50 \%$ and $90 \%$ acetone systems are shown in Figure 10. To emphasize their exponential decay, the $L(t)$ data are shown on a logarithmic scale.

As is seen, acetone molecules stay, on average, somewhat longer at the liquid surface than methanols, in spite of the fact that, within the surface layer, methanol molecules are located, on average, farther from the bulk liquid phase than acetones (see Fig. 6). Further, the mean surface residence time of acetone molecules slightly decreases with increasing acetone 
concentration (being about $20 \%$ smaller in neat acetone than in the $10 \%$ system), while this tendency is considerably weaker for methanol. There is a marked difference, however, between the survival probabilities and mean residence times of both components in the first and in the subsequent subsurface layers. Thus, the mean residence time of the molecules both in the second and in the third subsurface layer is always about an order of magnitude smaller than in the surface layer, being about $1.5 \mathrm{ps}$ for methanol and $2.5 \mathrm{ps}$ for acetone in every system simulated. This is also illustrated by the inset of Fig. 10, showing the $L(t)$ survival probabilities of both molecules in the first three subsurface layers of the equimolar system.

The fact that the $\tau$ values obtained in the second and third layers beneath the surface are comparable with the length of the $\Delta t$ time window used in the calculations indicate that, from a dynamical point of view, these layers are already part of the bulk liquid phase, as the effect of the proximity of the interface on the dynamics of the individual molecules is already lost in the second subsurface layer.

\subsection{Surface orientation}

Since the orientation of a rigid body of general shape (e.g., a molecule) relative to an external direction (e.g., the surface normal vector) can be described by two orientational parameters, the orientational statistics of the surface molecules relative to the macroscopic plane of the liquid surface can only be fully characterized by the bivariate joint distribution of two independent orientational quantities. ${ }^{56,57}$ We have shown that the angular polar coordinates $\vartheta$ and $\phi$ of the macroscopic surface normal vector, $\underline{X}$, (pointing from the liquid towards the vapor phase according to our convention) in a local Cartesian frame fixed to the individual molecules represent a sufficient choice of such an orientational parameter pair. However, concerning the fact that $\vartheta$ is an angle between two general spatial vectors, but $\phi$ is formed by two vectors restricted to lay in a given plane (i.e., the $x y$ plane of this local frame) by definition, uncorrelated orientation of the molecules with the surface results in a uniform bivariate distribution only if $\cos \vartheta$ and $\phi$ are chosen to be the orientational parameters. ${ }^{56,57}$

Here we define these local Cartesian frames in the following way. For acetone, its axis $x$ is perpendicular to the molecular plane, axis $z$ points along the main symmetry axis of the molecule, from the $\mathrm{O}$ to the central $\mathrm{C}$ atom, while axis $y$, being perpendicular to the above two axes, is parallel with the line joining the two $\mathrm{CH}_{3}$ groups. For methanol, axis $x$ points along the $\mathrm{CH}_{3}-\mathrm{O}$ bond from the $\mathrm{CH}_{3}$ group to the $\mathrm{O}$ atom, axis $y$ is still within the molecular plane, directed in such a way that the $y$ coordinate of the $\mathrm{H}$ atom is negative, and $z$ is the 
molecular normal axis. Due to the $\mathrm{C}_{2 \mathrm{v}}$ symmetry of the acetone, and planar symmetry of the methanol molecule, these frames are always chosen in such a way that, for acetone, the relation $0^{\circ} \leq \phi \leq 90^{\circ}$, while for methanol the relation $0^{\circ} \leq \vartheta \leq 90^{\circ}$ (and hence $0 \leq \cos \vartheta \leq 1$ ) holds. The definition of these local frames and of the polar angles $\vartheta$ and $\phi$ are illustrated in Figure $11 \mathrm{a}$ and $\mathrm{b}$ for acetone and methanol, respectively.

In order to investigate also the effect of the local curvature of the surface layer on the orientational preferences, we have divided the surface layer into three separate regions, marked by $\mathrm{A}, \mathrm{B}$ and $\mathrm{C}$, respectively, in the following way. Regions $\mathrm{A}$ and $\mathrm{C}$ cover the $X$ ranges at the vapor and liquid side of the density peak of the first layer, respectively, in which the mass density of the first layer is less than half of its maximum value, whereas region $B$ covers the $X$ range where the first layer mass density exceeds half of its maximum value. In this way, regions $\mathrm{A}$ and $\mathrm{C}$ typically cover the crests and troughs, i.e., surface portions of locally convex and concave curvature, respectively, of the molecularly rough intrinsic surface. The division of the surface layer into regions A, B and C is illustrated in Fig. 11.c.

The $P(\cos \vartheta, \phi)$ orientational maps of the surface acetone and methanol molecules are shown in Figures 12 and 13, respectively, as obtained in the entire surface layer as well as in its separate regions A, B and C of selected systems. As is seen, the preferred orientation of the surface acetone molecules correspond to the $\left[\cos \vartheta \approx 0.1 ; \phi=90^{\circ}\right]$ point of the orientational map in every system. In this orientation, denoted here as A1, the acetone molecule stays perpendicular to the macroscopic plane of the surface, $Y Z$, and its dipole vector, pointing along the $\mathrm{O}=\mathrm{C}$ double bond, is flatly directed towards the vapor phase, declining by no more than $5-10^{\circ}$ from the macroscopic plane of the interface, $Y Z$. This orientation is not only preferred in the entire surface layer, but also in its regions $\mathrm{B}$ and $\mathrm{C}$. However, in region $\mathrm{C}$ the peak of this orientation is shifted to somewhat smaller $\cos \vartheta$ values, being centered around $\cos \vartheta \approx-0.1$, indicating that acetone molecules located at the bottom of the troughs of the wavy surface prefer to point flatly towards the liquid phase by their $\mathrm{O}=\mathrm{C}$ double bond. Further, while the composition of the system has no visible effect on the strength of this orientation (i.e., height of the $P(\cos \vartheta, \phi)$ peak), the preference for this orientation is always markedly stronger in region $\mathrm{C}$ (i.e., at the bottom of the troughs of the wavy surface) than either in region B or in the entire surface layer. Furthermore, in region A, i.e., at the tips of the crests of the wavy surface, a different orientation, characterized by the $\cos \vartheta \approx 0.1$ and $\phi=0^{\circ}$ values, is preferred by the acetone molecules. In this orientation, marked here by A2, the dipolar vector (i.e., $\mathrm{O}=\mathrm{C}$ bond) of the molecule points again flatly towards the vapor phase, 
but the plane of the molecule adopts the orientation as close as possible to the parallel alignment with the macroscopic surface plane, YZ. Further, among these two preferences the former (i.e., the dipole vector being nearly parallel with the surface plane) is considerably stronger than the latter (i.e., the molecular plane being parallel with the surface plane), as the corresponding peak of $P(\cos \vartheta, \phi)$ is rather narrow along the $\cos \vartheta$, and broad along the $\phi$ axis. In other words, alignments A1 and A2 correspond to a $90^{\circ}$ flip of the acetone molecule around its main symmetry (i.e., $\mathrm{C}=\mathrm{O}$ ) axis, but somewhat smaller rotations can also contribute to the A2 peak. Both orientations A1 and A2 are illustrated in Figure 14.

The methanol molecules prefer the orientation corresponding to the $\cos \vartheta$ and $\phi$ values of 0 and $180^{\circ}$, respectively, in the entire surface layer as well as in all three of its separate regions. Similarly to acetone, neither this orientational preference itself nor its strength show a considerable dependence on the composition of the system. However, this preference is always markedly stronger in region A (i.e., at the tips of the crests) than in the other two regions and in the entire surface layer. In this orientation, marked by M1, the methanol molecule stays perpendicular to the macroscopic surface plane, $Y Z$, and its $\mathrm{O}-\mathrm{CH}_{3}$ bond sticks straight out to the vapor phase. It is also seen that this preference is markedly stronger in terms of the $\phi$ than of the $\cos \vartheta$ value. This means that the perpendicular alignment of the molecule to the $Y Z$ plane is only rather weakly preferred, while the $\mathrm{CH}_{3}$ group strongly prefers to stick as straight to the vapor phase as possible within the constraint of the alignment of the molecular plane. It should also be noted that in orientation M1 the dipole vector of the methanol molecule lays almost parallel with the macroscopic plane of the surface, $Y Z$, and this dipolar alignment is not even affected by some possible flip of the molecular plane. The orientation M1, along with A1 and A2 is also illustrated in Fig. 14.

All these results indicate that the interfacial arrangement of the molecules is primarily governed by dipole-dipole interactions. The fact that in all three preferred orientations the molecular dipole vectors lay preferentially almost parallel with the $Y Z$ plane, this preference being rather strong in every case, allows various (e.g., antiparallel, head-to-tail) dipolar arrangements of the neighboring molecules at the liquid surface. To confirm the dominance of the dipolar interaction over hydrogen bonding in the surface layer, we also calculated the average number of hydrogen bonds a surface molecule forms with another molecule of the surface layer. This value turned out to decrease continuously with increasing acetone mole fraction from the value of 1.21, characteristic of neat methanol, to zero. However, in spite of its secondary importance, the observed orientational preferences also reflect the hydrogen 
bond formation between surface methanol and acetone molecules. Thus, a methanol molecule of orientation $\mathrm{M} 1$ can easily donate its $\mathrm{H}$ atom to an acetone molecule of orientation $\mathrm{A} 1$, as illustrated in Fig. 14. Considering the fact that in orientation M1 the methanol O-H bond is slightly inward oriented, the formation of such a hydrogen bond requires the acetone molecule being somewhat closer to the bulk liquid phase along the macroscopic surface normal axis, $X$, than the methanol (see Fig. 14). This fact explains the enhanced preference of the methanol and acetone molecules for orientations $\mathrm{M} 1$ and $\mathrm{A} 1$ in regions $\mathrm{A}$ and $\mathrm{C}$ of the surface layer, respectively, and also our previous observation that surface methanol molecules prefer, on average, being slightly closer to the vapor phase than surface acetones (see Fig. 6).

Finally, it should be noted that the $P(\cos \vartheta, \phi)$ orientational maps calculated in the second and third subsurface layers as well as in their separate regions A, B and C always turned out to be completely uniform, indicating that the effect of the proximity of the interface on the orientation of the molecules also vanishes beyond the first molecular layer.

\section{Summary and conclusions}

In this paper we presented a detailed analysis of the liquid-vapor interface of acetonemethanol mixtures of various compositions, on the basis of molecular dynamics simulations performed using a potential model combination that can very well describe the mixing properties of these compounds, ${ }^{7}$ and of the determination of the intrinsic liquid surface by means of the ITIM method. The obtained results have revealed that, similarly to the bulk liquid phase, ${ }^{4-7}$ the two molecules tend to self-associate also within the surface layer. This self-association behavior can be explained by the fact that the full miscibility of the two components is governed by the entropy term of the ideal mixing, while the energy of mixing is positive. ${ }^{7}$ Interestingly, the width of the surface layer has turned out to be the largest at the composition where this energy of mixing is the largest, probably because of the non-perfect packing of these relatively large self-associates. On the other hand, no microscopic separation of the two components has been observed along the macroscopic surface normal axis, as none of them has shown considerable tendency for surface adsorption, although, within the surface layer, methanols have been found to stay, on average, somewhat closer to the vapor phase than acetones. The similar surface affinity of the two molecules has also resulted in the composition independence of the molecular scale roughness of the liquid surface. 
Acetone molecules have turned out to stay, on average, somewhat (i.e., by about 50\%) longer at the liquid surface than methanols. Further, the mean surface residence time of both molecules has been found to decrease with increasing acetone mole fraction, probably due to the decreasing extent of hydrogen bonding within the surface layer. In all the preferred surface orientations of both molecules, the molecular dipole vectors lay almost parallel with the macroscopic plane of the surface, pointing flatly (i.e., by $10-20^{\circ}$ ) away from the liquid phase. This finding, together with the relatively small number of hydrogen bonds formed by two surface molecules indicate that the surface orientation of the molecules is primarily governed by dipolar interactions. Although hydrogen bonds play only a secondary role in this respect, methanol-acetone hydrogen bonds within the surface layer also have some influence on the surface structure. Due to the orientational preferences of the molecules the formation of such a hydrogen bond assumes that, among the two H-bonding partners, the methanol molecule stays closer to the vapor phase. This is reflected both in the enhanced strength of the observed orientational preferences at the ripples of the molecularly rugged surface for methanols and at its troughs for acetone, and also in the fact that within the surface layer methanol molecules prefer to stay, on average, somewhat closer to the vapor phase than acetones.

Finally all of our results indicate that the influence of the vapor phase on the properties of the nearby liquid phase is lost beyond the first molecular layer, hence, the second molecular layer beneath the liquid surface can already be regarded as part of the bulk liquid phase in every respect.

\section{Acknowledgements}

This work has been supported by the Hungarian OTKA Foundation under Project No. OTKA 104234, by the Hungarian-French Intergovernmental Science and Technology Program (BALATON) under project No. TéT_12_FR-1-2013-0013. The Centre de Ressources Informatiques (CRI) de l'Université de Lille, and the Centre de Ressource Informatique de Haute-Normandie (CRIHAN) are thankfully acknowledged for the CPU time allocation. P. J. is a Szentágothai János fellow of Hungary, supported by the European Union, co-financed by the European Social Fund in the framework of TÁMOP 4.2.4.A/2-11/1-20120001 “National Excellence Program” under grant number A2-SZJÖ-TOK-13-0030. 


\section{References}

1. D. S. Venables and C. A. Schmuttenmaer, J. Chem. Phys., 2000, 113, 3249.

2. W. Yi, C. Wang H. Li and S. Han, J. Chem. Eng. Data, 2005, 50, 1837.

3. G. Modla and P. Lang, Ind. Eng. Chem. Res., 2010, 49, 3785.

4. G. Kamath, G. Georgiev and J. J. Potoff, J. Phys. Chem. B, 2005, 109, 19463.

5. A. Perera, L. Zoranić, F. Sokolić and R. Mazighi, J. Mol. Liquids, 2011, 159, 52.

6. A. Idrissi, K. Polok, W. Gadomski, I. Vyalov, A. Agapov, M. Kiselev, M. Barj and P. Jedlovszky, Phys. Chem. Chem. Phys., 2012, 14, 5979.

7. A. Idrissi, K. Polok, M. Barj, B. Marekha, M. Kiselev and Jedlovszky, P. J. Phys. Chem. B, 2013, 117, 16157.

8. L. B. Pártay, Gy. Hantal, P. Jedlovszky, Á. Vincze and G. Horvai, J. Comp. Chem., 2008, 29, 945.

9. Gy. Hantal, M. Darvas, L. B. Pártay, G. Horvai and P. Jedlovszky, J. Phys.: Condens. Matter, 2010, 22, 284112.

10. L. B. Pártay, P. Jedlovszky, Á. Vincze and G. Horvai, J. Phys. Chem. B., 2008, 112, 5428.

11. L. B. Pártay, P. Jedlovszky and G. Horvai, J. Phys. Chem. C., 2009, 113, 18173.

12. K. Pojják, M. Darvas, G. Horvai, and P. Jedlovszky, J. Phys. Chem. C., 2010, 114, 12207.

13. L. B. Pártay, G. Horvai, and P. Jedlovszky, J. Phys. Chem. C., 2010, 114, 21681.

14. P. Linse, J. Chem. Phys., 1987, 86, 4177.

15. I. Benjamin, J. Chem. Phys., 1992, 97, 1432.

16. M. Jorge and M. N. D. S. Cordeiro, J. Phys. Chem. C., 2007, 111, 17612.

17. A. S. Pandit, D. Bostick and M. L. Berkowitz, J. Chem. Phys., 2003, 119, 2199.

18. E. Chacón and P. Tarazona, Phys Rev. Letters, 2003, 91, 166103.

19. E. Chacón and P. Tarazona, J. Phys.: Condens. Matter, 2005, 17, S3493.

20. J. Chowdhary and B. M. Ladanyi, J. Phys. Chem. B., 2006, 110, 15442.

21. M. Jorge, P. Jedlovszky and M. N. D. S. Cordeiro, J. Phys. Chem. C., 2010, 114, 11169.

22. M. Mezei, J. Mol. Graphics Modell., 2003, 21, 463.

23. A. P. Wilard and D. Chandler, J. Phys. Chem. B., 2010, 114, 1954. 
24. M. Sega, S. S. Kantorovich, P. Jedlovszky, and M. Jorge, J. Chem. Phys., 2013, 138, 044110 .

25. M. Sega, G. Horvai and P. Jedlovszky, Langmuir, 2014, 30, 2969.

26. M. Sega, G. Horvai and P. Jedlovszky, J. Chem. Phys., 2014, 141, 054707.

27. L. B. Pártay, G. Horvai, and P. Jedlovszky, Phys. Chem. Chem. Phys., 2008, 10, 4754.

28. G. Hantal, P. Terleczky, G. Horvai, L. Nyulászi and P. Jedlovszky, J. Phys. Chem. C, 2009, 113, 19263.

29. M. Darvas, L. B. Pártay, P. Jedlovszky and G. Horvai, J. Mol. Liquids, 2010, 153, 88.

30. M. Darvas, K. Pojják, G. Horvai and P. Jedlovszky, J. Chem. Phys., 2010, 132, 134701.

31. M. Darvas, T. Gilányi and P. Jedlovszky, J. Phys. Chem. B, 2011, 115, 933.

32. Gy. Hantal, M. N. D. S. Cordeiro and M. Jorge, Phys. Chem. Chem. Phys., 2011, 13, 21230.

33. M. Lísal, Z. Posel and P. Izák, Phys. Chem. Chem. Phys., 2012, 14, 5164.

34. Gy. Hantal, I. Voroshylova, M. N. D. S. Cordeiro and M. Jorge, Phys. Chem. Chem. Phys., 2012, 14, 5200.

35. M. Lísal and P. Izák, J. Chem. Phys., 2013, 139, 014704.

36. N. Abrankó-Rideg, M. Darvas, G. Horvai and P. Jedlovszky, J. Phys. Chem. B, 2013, 117, 8733.

37. B. Fábián, M. Szőri and P. Jedlovszky, J. Phys. Chem. C, 2014, 118, 21469.

38. P. Jedlovszky, B. Jójárt and G. Horvai, Mol. Phys., in press. DOI: $10.1080 / 00268976.2014 .968227$

39. M. Darvas, M. Jorge, M. N. D. S. Cordeiro, S. S. Kantorovich, M. Sega and P. Jedlovszky, J. Phys. Chem. B, 2013, 117, 16148.

40. M. Darvas, M. Jorge, M. N. D. S. Cordeiro and P. Jedlovszky, J. Mol. Liquids, 2014, 189, 39.

41. B. Chen, J. J. Potoff and J. I. Siepmann, J. Phys. Chem. B, 2001, 105, 3093.

42. M. P. Allen and D. J. Tildesley, Computer Simulation of Liquids, Clarendon Press, Oxford, 1987.

43. U. Essman, L. Perera, M. L. Berkowitz, T. Darden, H. Lee and L. G. Pedersen, J. Chem. Phys., 1995, 103, 8577.

44. B. Hess, J. Chem. Theory Comput., 2008, 4, 116.

45. B. Hess, C. Kutzner, D. van der Spoel, and E. Lindahl, J. Chem. Theory Comput., 2008, 4, 435. 
46. S. Nosé, Mol. Phys., 1984, 52, 255.

47. W. G. Hoover, Phys. Rev. A, 1985, 31, 1695.

48. Y. Mlyano and W. Hayduk, J. Chem. Eng. Data, 1986, 31, 81.

49. CRC Handbook of Chemistry and Physics, ed.: D. R. Lide, CRC Press, Boca Raton, 1997-1998.

50. J. Chowdhary and B. M. Ladanyi, Phys. Rev. E, 2008, 77, 031609.

51. G. Voronoi, J. Reine Angew. Math., 1908, 1908, 198.

52. N. N. Medvedev, The Voronoi-Delaunay Method in Srtuctural Studies of Noncrystalline Systems, SB RAS, Novosibirsk, 2000.

53. A. Okabe, B. Boots, K. Sugihara and S. N. Chiu, Spatial Tessellations: Concepts and Applications of Voronoi Diagrams, John Wiley \& Sons, Inc., Chichester, 2000.

54. L. Zaninetti, Phys. Lett. A, 1992, 165, 143.

55. A. Idrissi, P. Damay, K. Yukichi and P. Jedlovszky, J. Chem. Phys., 2008, 129, 164512 .

56. P. Jedlovszky, Á. Vincze and G. Horvai, J. Chem. Phys., 2002, 117, 2271.

57. P. Jedlovszky, Á. Vincze and G. Horvai, Phys. Chem. Chem. Phys., 2004, 6, 1874 


\section{Tables}

Table 1. Interaction parameters of the molecular models used

\begin{tabular}{ccccc}
\hline \hline molecule & interaction site & $q / \mathrm{e}$ & $\left(\varepsilon / k_{\mathrm{B}}\right) / \mathrm{K}$ & $\sigma / \AA$ \\
\hline \multirow{3}{*}{ acetone $^{\mathrm{a}}$} & $\mathrm{CH}_{3}$ & -0.049 & 98.0 & 3.75 \\
& $\mathrm{C}$ & 0.662 & 27.0 & 3.82 \\
& $\mathrm{O}$ & -0.564 & 79.0 & 3.05 \\
methanol $^{\mathrm{b}}$ & $\mathrm{CH}_{3}$ & 0.265 & 98.0 & 3.75 \\
& $\mathrm{O}$ & -0.700 & 93.0 & 3.02 \\
\hline \hline
\end{tabular}

${ }^{\mathrm{a}}$ Ref. 4.

${ }^{\mathrm{b}}$ Ref. 41. 
Table 2. Calculated properties of the first three molecular layers of the systems simulated

\begin{tabular}{|c|c|c|c|c|c|c|c|}
\hline \multirow{2}{*}{ layer } & \multirow{2}{*}{$x_{\mathrm{ac}}$} & \multirow{2}{*}{$\delta / \AA ̊$} & \multirow{2}{*}{$X_{\mathrm{C}} / \AA ̊$} & \multirow{2}{*}{$\xi$} & \multirow{2}{*}{$a / \AA ̊$} & \multicolumn{2}{|c|}{$\tau / \mathrm{ps}$} \\
\hline & & & & & & methanol & acetone \\
\hline \multirow{11}{*}{$\begin{array}{l}\text { first } \\
\text { layer }\end{array}$} & 0.0 & 5.93 & 26.07 & 1.30 & 4.55 & 22.0 & - \\
\hline & 0.1 & 6.01 & 28.03 & 1.47 & 5.21 & 23.5 & 36.0 \\
\hline & 0.2 & 6.02 & 30.24 & 1.61 & 5.50 & 23.0 & 35.7 \\
\hline & 0.3 & 6.01 & 32.43 & 1.68 & 5.65 & 22.3 & 34.1 \\
\hline & 0.4 & 6.20 & 34.66 & 1.77 & 5.98 & 21.7 & 32.4 \\
\hline & 0.5 & 6.22 & 36.90 & 1.60 & 5.51 & 22.0 & 32.0 \\
\hline & 0.6 & 6.16 & 39.14 & 1.61 & 5.58 & 21.8 & 31.8 \\
\hline & 0.7 & 6.91 & 41.38 & 1.76 & 5.99 & 22.0 & 31.6 \\
\hline & 0.8 & 6.85 & 43.73 & 1.66 & 5.56 & 22.0 & 30.6 \\
\hline & 0.9 & 6.69 & 45.90 & 1.60 & 5.43 & 21.8 & 30.4 \\
\hline & 1.0 & 6.26 & 48.15 & 1.58 & 5.35 & - & 30.3 \\
\hline \multirow{11}{*}{$\begin{array}{c}\text { second } \\
\text { layer }\end{array}$} & 0.0 & 5.69 & 21.89 & 0.80 & 3.54 & 2.1 & - \\
\hline & 0.1 & 5.82 & 23.71 & 0.79 & 3.58 & 2.0 & 3.8 \\
\hline & 0.2 & 5.87 & 25.73 & 0.83 & 3.52 & 1.9 & 3.1 \\
\hline & 0.3 & 5.89 & 27.77 & 0.85 & 3.48 & 1.9 & 3.0 \\
\hline & 0.4 & 6.05 & 29.85 & 0.85 & 3.53 & 1.8 & 2.6 \\
\hline & 0.5 & 6.06 & 31.95 & 0.85 & 3.57 & 1.7 & 2.6 \\
\hline & 0.6 & 6.01 & 34.10 & 0.86 & 3.50 & 1.7 & 2.5 \\
\hline & 0.7 & 6.71 & 36.22 & 0.86 & 3.54 & 1.6 & 2.3 \\
\hline & 0.8 & 6.73 & 38.51 & 0.87 & 3.51 & 1.6 & 2.1 \\
\hline & 0.9 & 6.48 & 40.56 & 0.86 & 3.49 & 1.6 & 2.2 \\
\hline & 1.0 & 6.01 & 42.74 & 0.86 & 3.49 & - & 2.2 \\
\hline \multirow{11}{*}{$\begin{array}{l}\text { third } \\
\text { layer }\end{array}$} & 0.0 & 5.47 & 17.73 & 0.71 & 3.45 & 2.4 & - \\
\hline & 0.1 & 5.66 & 19.39 & 0.73 & 3.50 & 2.3 & 3.4 \\
\hline & 0.2 & 5.74 & 21.24 & 0.77 & 3.46 & 2.2 & 3.5 \\
\hline & 0.3 & 5.79 & 23.13 & 0.80 & 3.43 & 2.1 & 3.0 \\
\hline & 0.4 & 5.93 & 25.09 & 0.80 & 3.46 & 1.9 & 2.7 \\
\hline & 0.5 & 5.97 & 27.05 & 0.81 & 3.51 & 1.8 & 2.6 \\
\hline & 0.6 & 5.93 & 29.11 & 0.81 & 3.45 & 1.9 & 2.4 \\
\hline & 0.7 & 6.58 & 31.12 & 0.81 & 3.46 & 1.6 & 2.3 \\
\hline & 0.8 & 6.75 & 33.35 & 0.81 & 3.43 & 1.7 & 2.3 \\
\hline & 0.9 & 6.38 & 35.33 & 0.80 & 3.43 & 1.7 & 2.2 \\
\hline & 1.0 & 5.90 & 37.44 & 0.80 & 3.42 & - & 2.2 \\
\hline
\end{tabular}




\section{Figure legend}

Fig. 1. Instantaneous equilibrium snapshot of the equimolar system, as taken out from the simulation. The molecules belonging to the first, second and third molecular layers beneath the surface are shown by red, green and blue colors, respectively, while the molecules located beneath the third molecular layer are marked by grey color. Acetone and methanol molecules are denoted by the darker and lighter shades of the colors. The snapshot only shows the $\mathrm{X}>0 \AA$ half of the basic simulation box. $\underline{X}$ is the macroscopic surface normal vector, pointing from the liquid to the vapor phase.

Fig. 2. Number density profiles of the methanol (top panel) and acetone (second panel) molecules as well as mass density profiles of the entire system (third panel) and its surface layer (bottom panel) along the surface normal axis, $X$, of the systems containing $0 \%$ (full circles), 10\% (red solid lines), 30\% (green dashed lines), 50\% (blue dash-dotted lines), 70\% (orange dash-dot-dotted lines), 90\% (magenta dotted lines) and 100\% (open circles) acetone. All profiles shown are symmetrized over the two liquid-vapor interfaces present in the basic box.

Fig. 3. Acetone mole fraction in the first (red squares), second (green circles) and third (blue triangles) molecular layer beneath the liquid surface as a function of the bulk phase acetone mole fraction. For reference, the straight line corresponding to the bulk phase acetone mole fraction is also shown by a solid line. Error bars are smaller than the symbols.

Fig. 4. Mass density profile of the entire system simulated (solid lines) as well as of the first (filled red circles), second (open blue circles) and third molecular layer (green asterisks) beneath the liquid surface along the surface normal axis, $X$, in the systems containing $10 \%$ (top panel), 40\% (second panel), 60\% (third panel) and 90\% (bottom panel) acetone. All profiles shown are symmetrized over the two liquid-vapor interfaces present in the basic box. 
Fig. 5. Dependence of the width parameter, $\delta$, of the first (black squares), second (red circles) and third (green triangles) molecular layers beneath the liquid surface on the bulk phase acetone mole fraction. The inset shows the dependence of the average separation, $\Delta X_{\mathrm{c}}$, of the first and second (full circles) and of the second and third (open circles) molecular layers beneath the liquid surface on the bulk phase acetone mole fraction. For the definition of the quantities $\delta$ and $\Delta X_{\mathrm{c}}$, see the text.

Fig. 6. Number density profile of the acetone (solid curves) and methanol (dashed curves) molecules in the surface layer of the systems containing 10\% (top panel), 40\% (second panel), $60 \%$ (third panel) and 90\% (bottom panel) acetone. The positions of the acetone and methanol molecules are represented by their central $\mathrm{C}$ atom and $\mathrm{CH}_{3}$ group, respectively. All profiles shown are symmetrized over the two liquid-vapor interfaces present in the basic box. The scales at the left and right side of the graphs refer to the methanol and acetone number densities, respectively.

Fig. 7. Average normal distance of two surface points, $\bar{d}$, as a function of their lateral distance, $l$, in the systems containing $10 \%$ (red solid lines), 30\% (green dashed lines), 50\% (blue dash-dotted lines), 70\% (orange dash-dot-dotted lines), and 90\% (magenta dotted lines) acetone. The inset shows this roughness curve in the first (solid line), second (full circles) and third (open circles) molecular layer of the equimolar system.

Fig. 8. Voronoi polygon area distribution of the surface molecules, projected to the macroscopic plane of the surface, $Y Z$, in the systems containing $0 \%$ (full circles), $10 \%$ (red solid lines), 30\% (green dashed lines), 50\% (blue dash-dotted lines), 70\% (orange dash-dotdotted lines), 90\% (magenta dotted lines) and 100\% (open circles) acetone, when all molecules have been considered in the Voronoi analysis (top panel), only the acetone molecules have been considered while methanols have been disregarded (middle panel), and only the methanol molecules have been considered while acetones have been disregarded (bottom panel). To emphasize the exponential decay of some curves, the plots are shown on a logarithmic scale. 
Fig. 9. Instantaneous equilibrium snapshot of the surface layer of the systems containing $20 \%$ (left), $50 \%$ (middle) and $80 \%$ (right) acetone, where the centers of the molecules are projected to the macroscopic plane of the surface, $Y Z$. Acetone and methanol molecules are represented by green and red balls, respectively.

Fig. 10. Survival probability of the acetone (top panel) and methanol (bottom panel) molecules in the surface layer of the systems containing 10\% (squares), 50\% (circles) and 90\% (triangles) acetone. The inset shows the survival probability of the acetone and methanol molecules in the first (black circles), second (red circles) and third (blue circles) molecular layers beneath the surface of the equimolar system. Filled and open symbols always correspond to the acetone and methanol molecules, respectively. To emphasize the exponential decay of the survival probabilities, the plots are shown on a logarithmic scale.

Fig. 11. Definition of the local Cartesian frames fixed to the individual (a) acetone and (b) methanol molecules, and of the polar angles $\vartheta$ and $\phi$ describing the orientation of the surface normal vector, $\underline{X}$, pointing, by our convention, from the liquid to the vapor phase, in these frames. (c) Illustration of the division of the surface layer into separate regions $\mathrm{A}, \mathrm{B}$ and $\mathrm{C}$ according to the mass density profile of the surface molecular layer.

Fig. 12. Orientational maps of the surface acetone molecules in the systems containing $10 \%$ (top row), 30\% (second row), 50\% (third row), 70\% (fourth row), 90\% (fifth row) and 100\% (bottom row) acetone. The first column corresponds to the entire first molecular layer beneath the surface, while the second, third and fourth column correspond to its separate regions A, B and $\mathrm{C}$, respectively. Lighter shades of grey denote higher probabilities. The peaks corresponding to the different preferred acetone orientations are marked by A1 and A2.

Fig. 13. Orientational maps of the surface methanol molecules in the systems containing $0 \%$ (top row), $10 \%$ (second row), 30\% (third row), 50\% (fourth row), 70\% (fifth row) and 90\% (bottom row) acetone. The first column corresponds to the entire first molecular layer beneath the surface, while the second, third and fourth column correspond to its separate regions $\mathrm{A}, \mathrm{B}$ and $\mathrm{C}$, respectively. Lighter shades of grey denote higher probabilities. The peaks corresponding to the preferred methanol orientation are marked by M1. 
Fig. 14. (a) Illustration of the preferred orientations of the acetone and methanol molecules in the surface layer of their mixtures. $\underline{X}$ is the macroscopic surface normal vector, pointing from the liquid to the vapor phase. (b) Illustration of the typical arrangement of a hydrogen bonded surface methanol-acetone pair. The H-bond is marked by a dotted line, the molecular dipole vectors are indicated by arrows. The solid line shows the molecularly wavy liquid surface. $\mathrm{C}$ and $\mathrm{O}$ atoms are indicated by grey and red colors, respectively. 
Figure 1.

Idrissi et al.

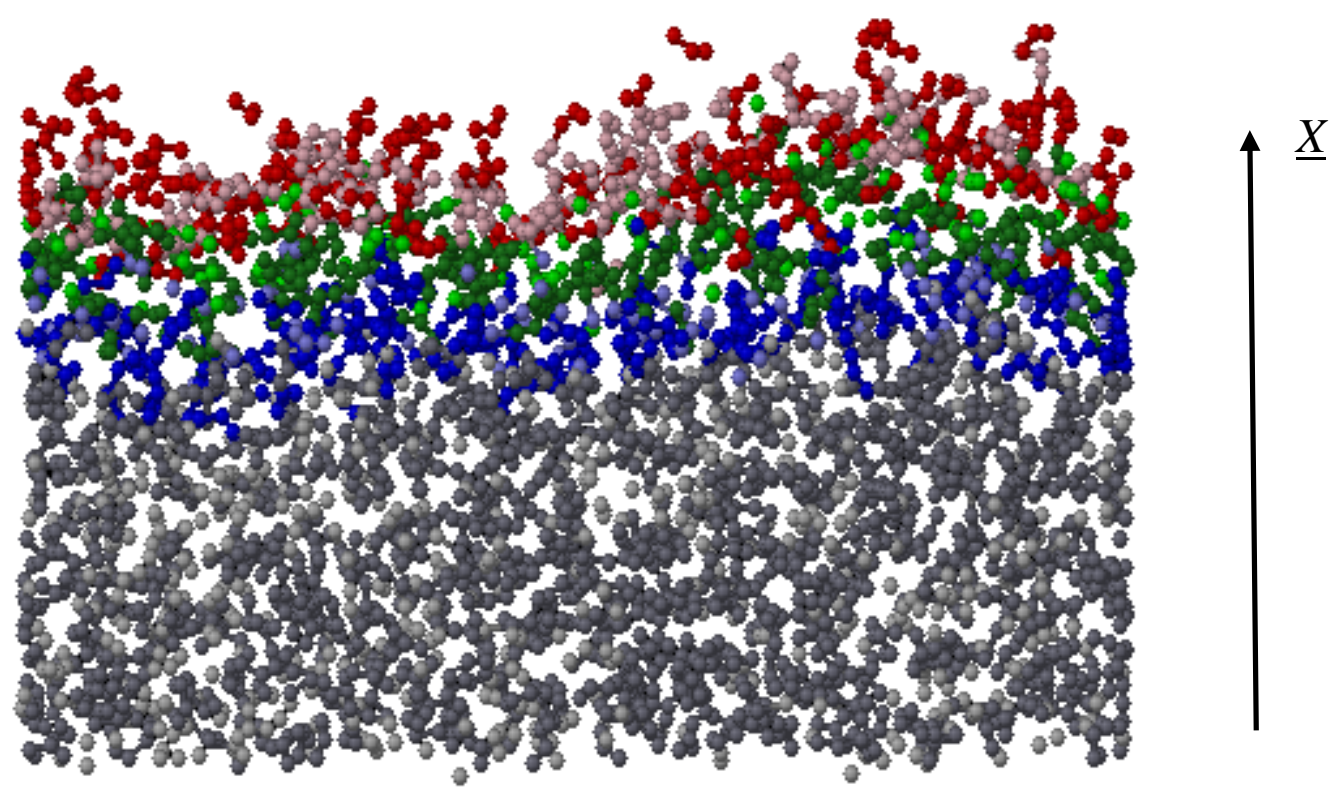


Figure 2.

Idrissi et al.

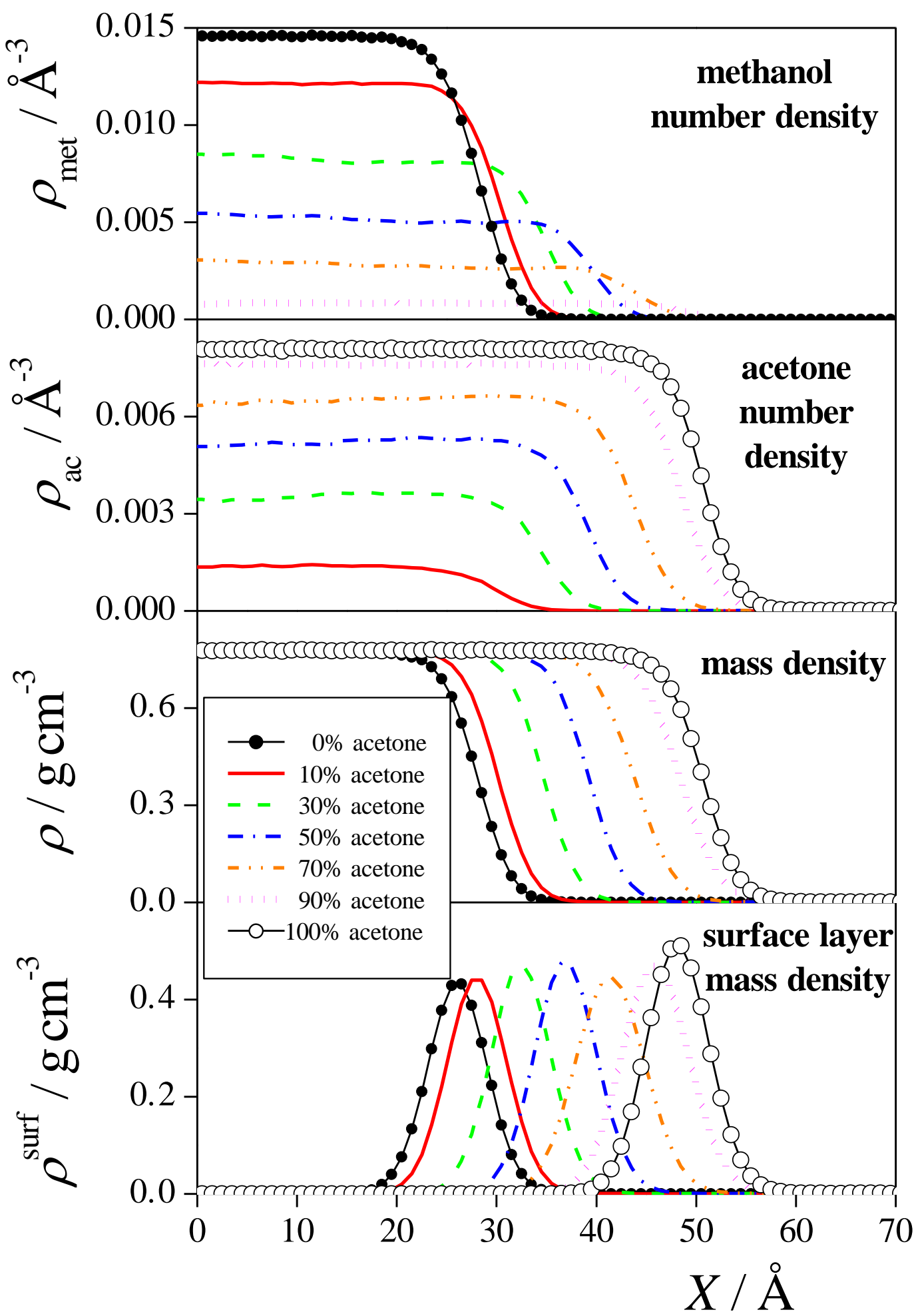


Figure 3.

Idrissi et al.

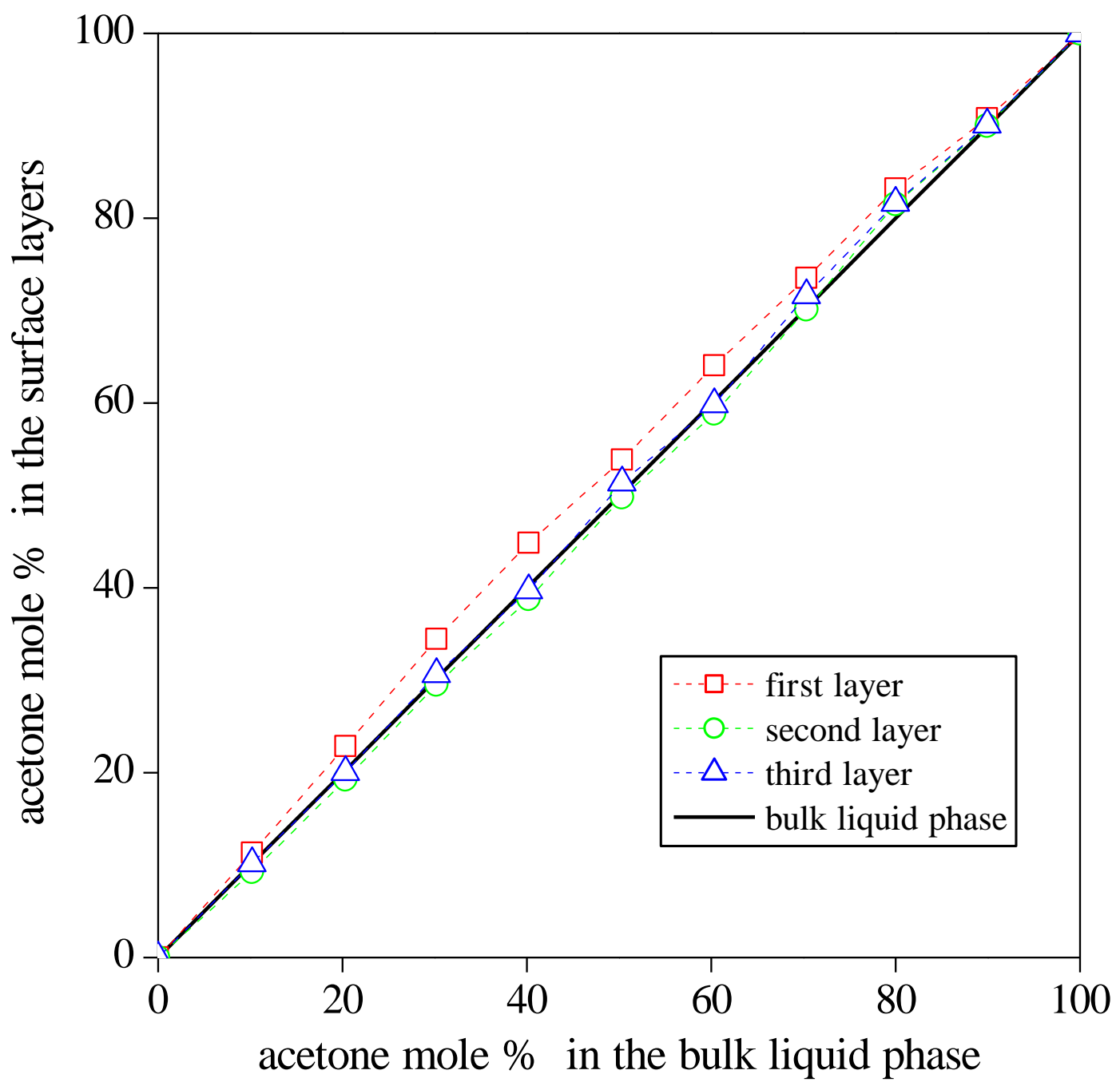


Figure 4.

Idrissi et al.

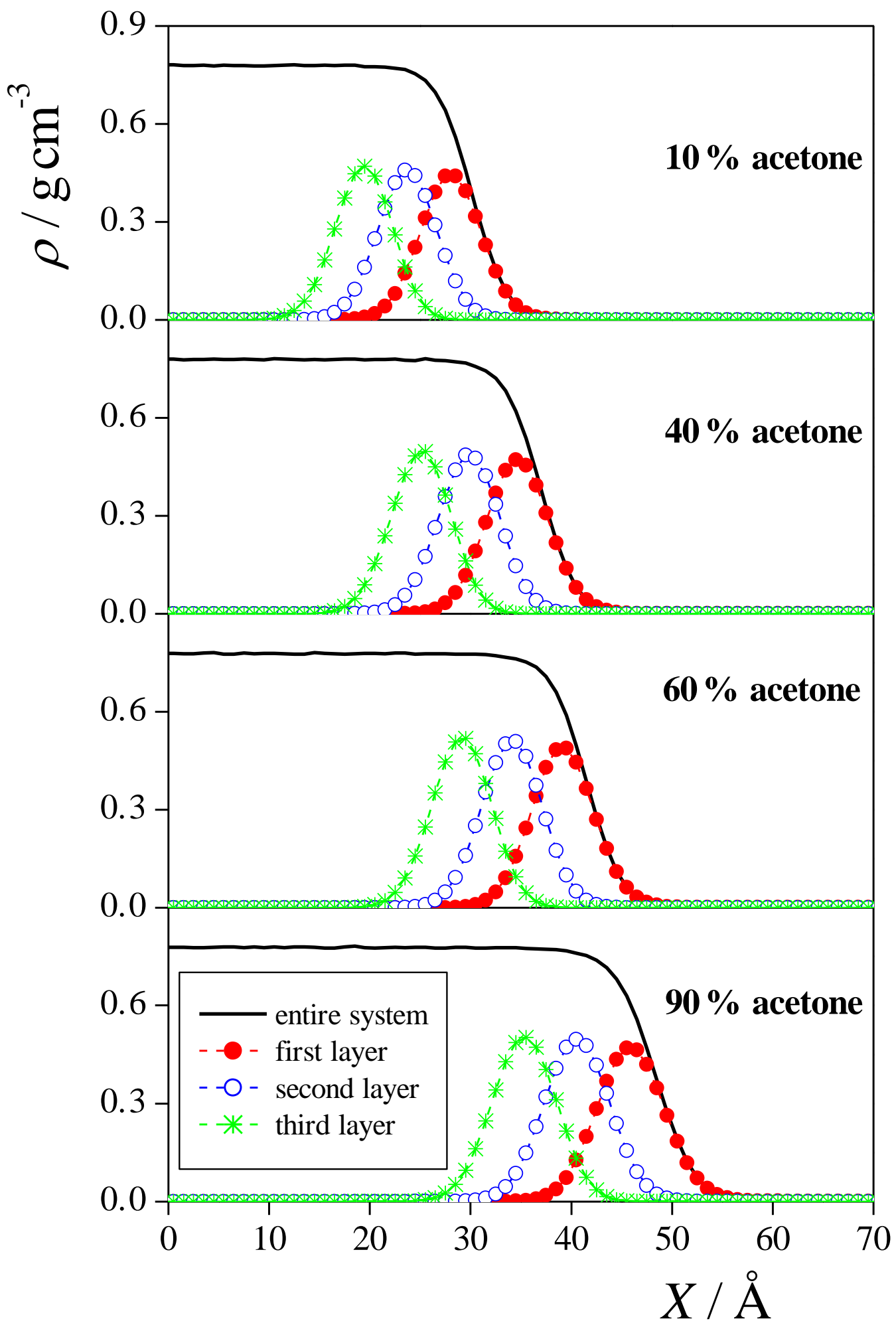


Figure 5.

Idrissi et al.

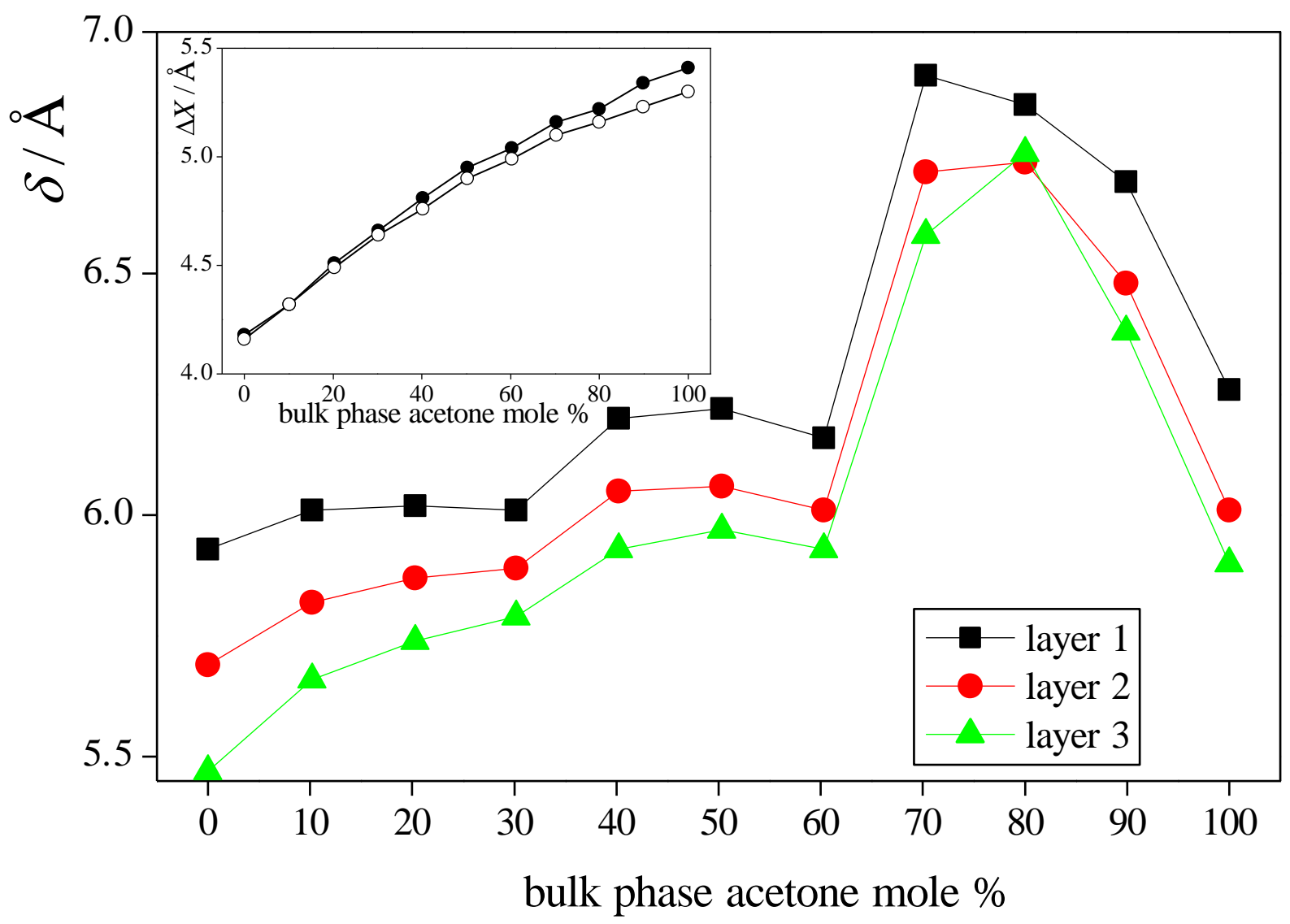


Figure 6.

Idrissi et al.

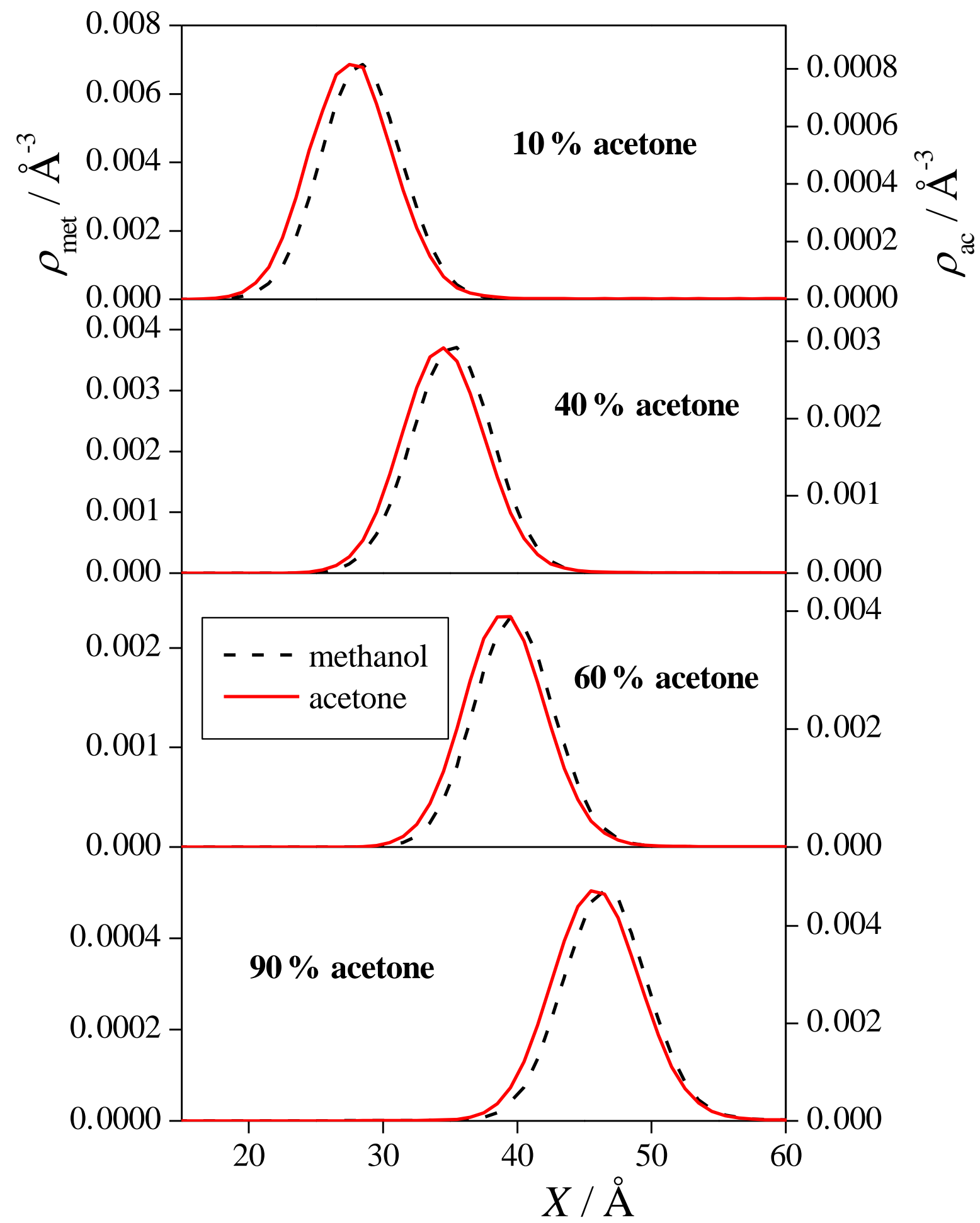


Figure 7.

Idrissi et al.

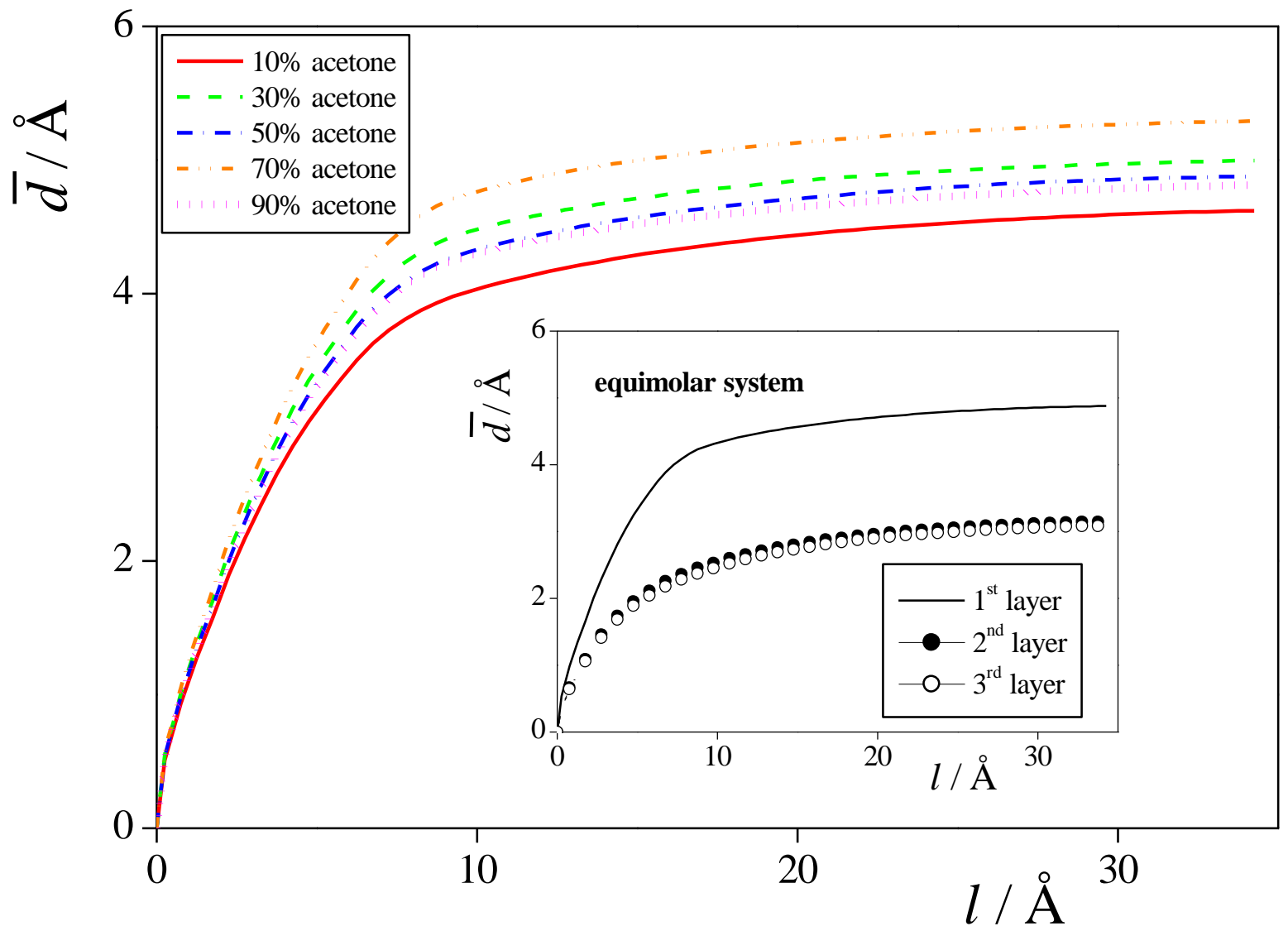


Figure 8.

Idrissi et al.

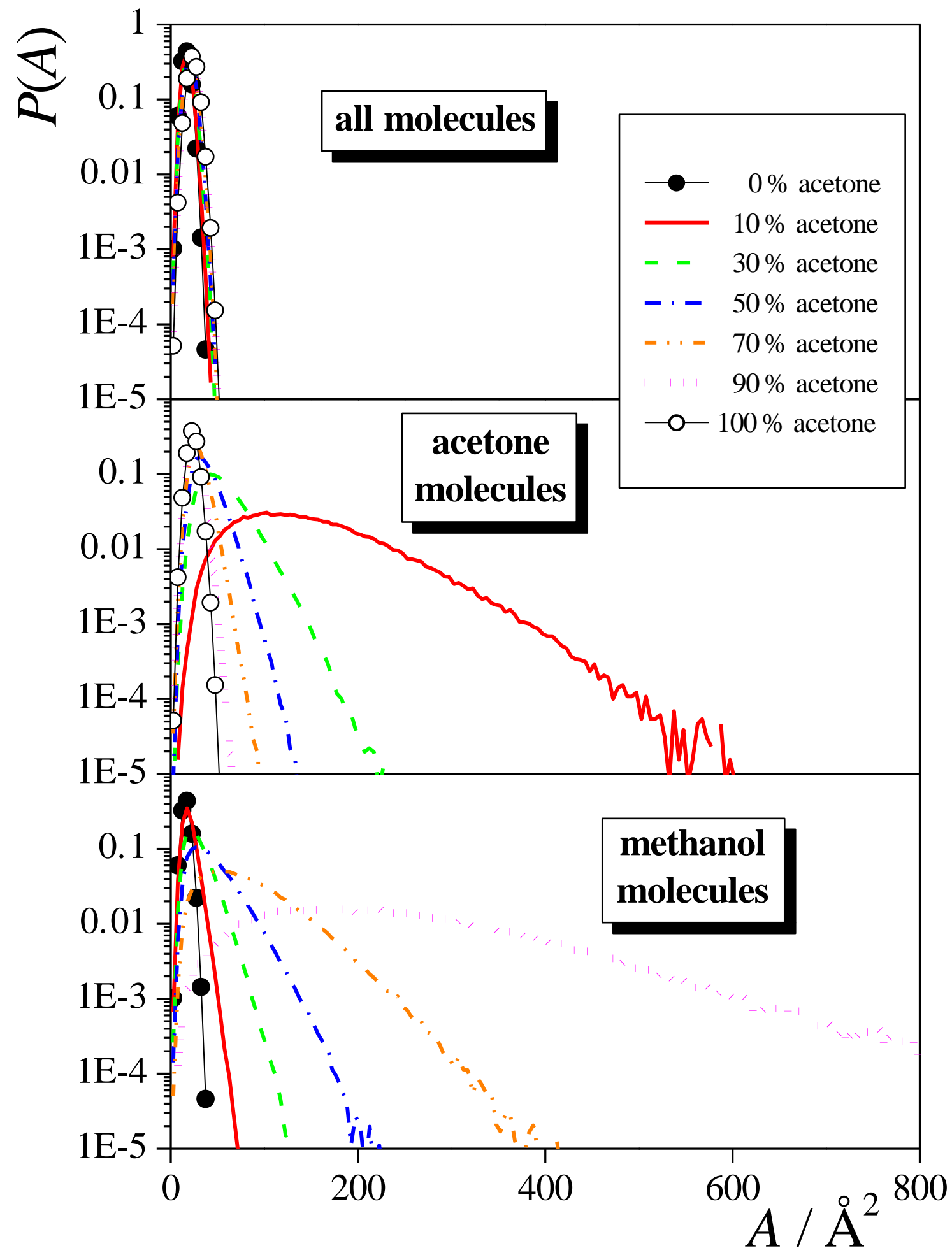


Figure 9.

Idrissi et al.

$20 \%$ acetone system

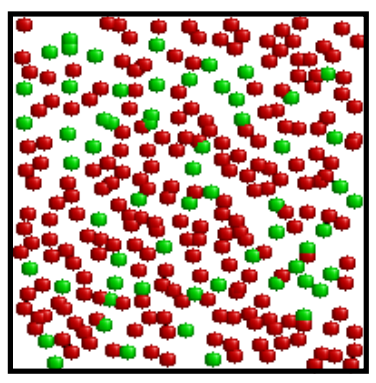

$50 \%$ acetone system

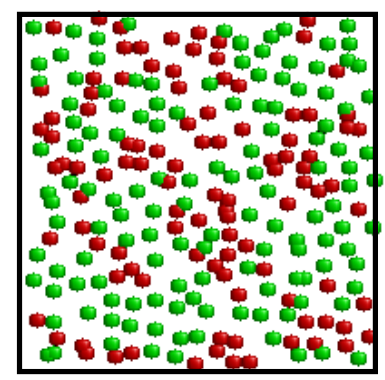

$80 \%$ acetone system

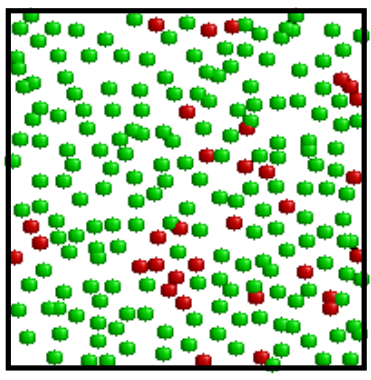


Figure 10.

Idrissi et al.

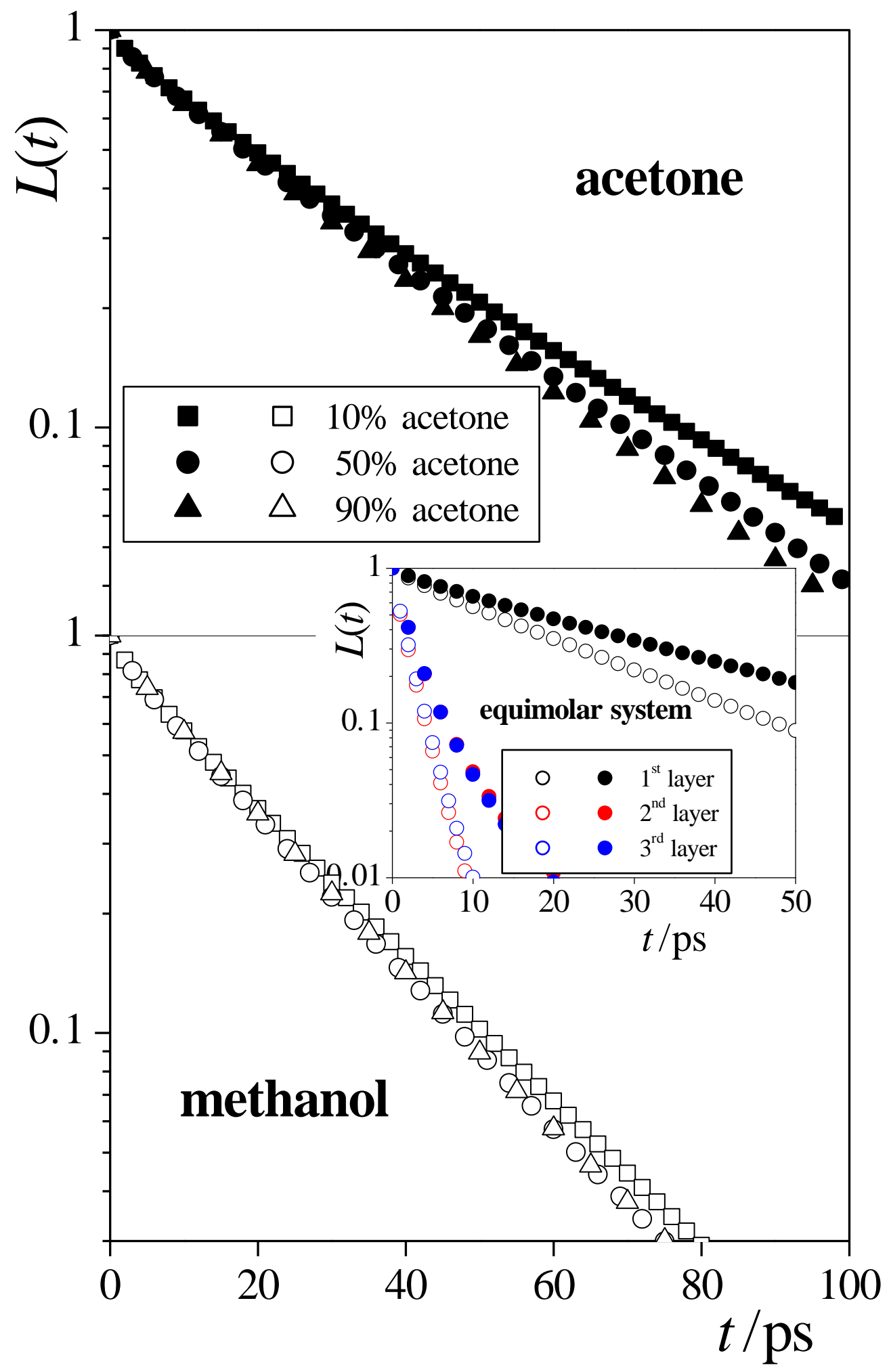


Figure 11.

Idrissi et al.

(a)
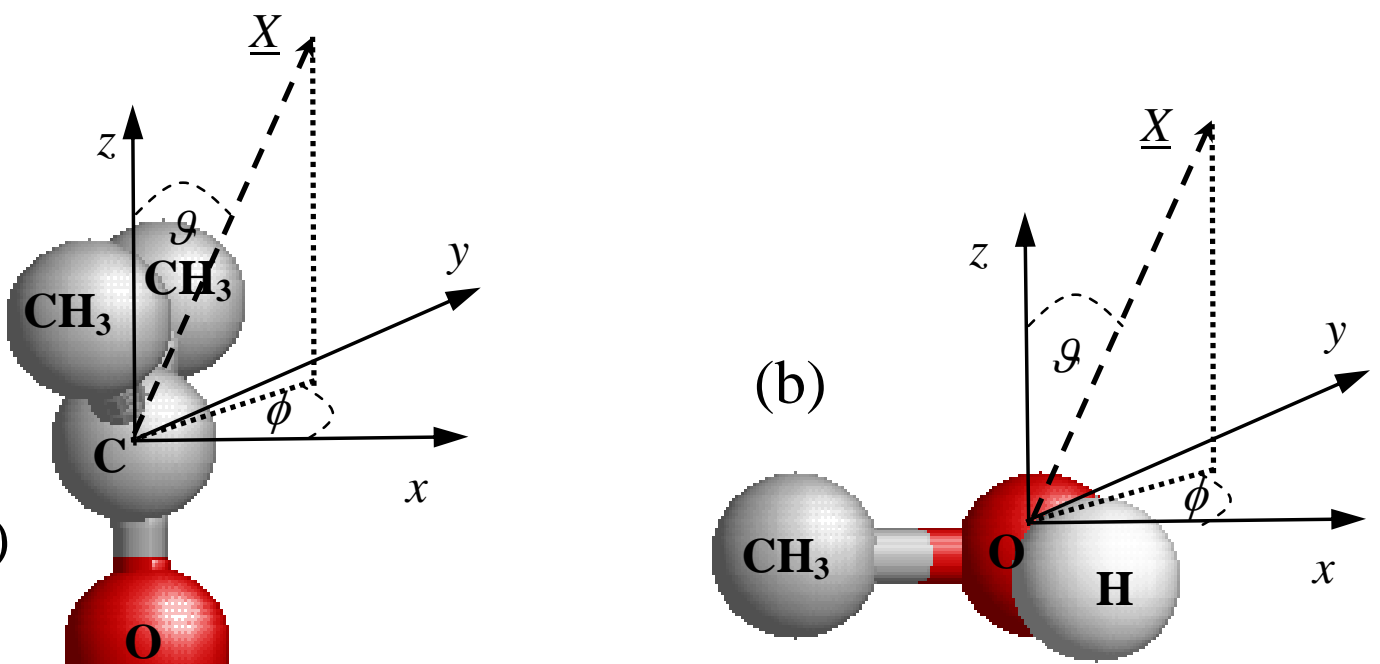

(c)

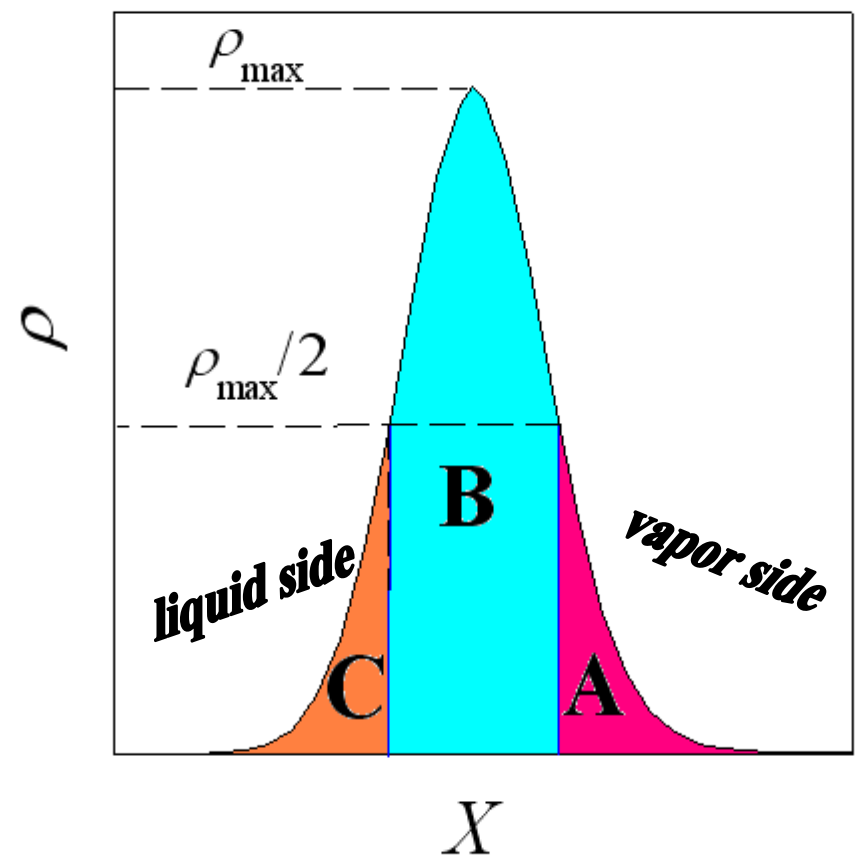


Figure 12.

Idrissi et al.

entire surface region $A$

region $B$

region $\mathbf{C}$
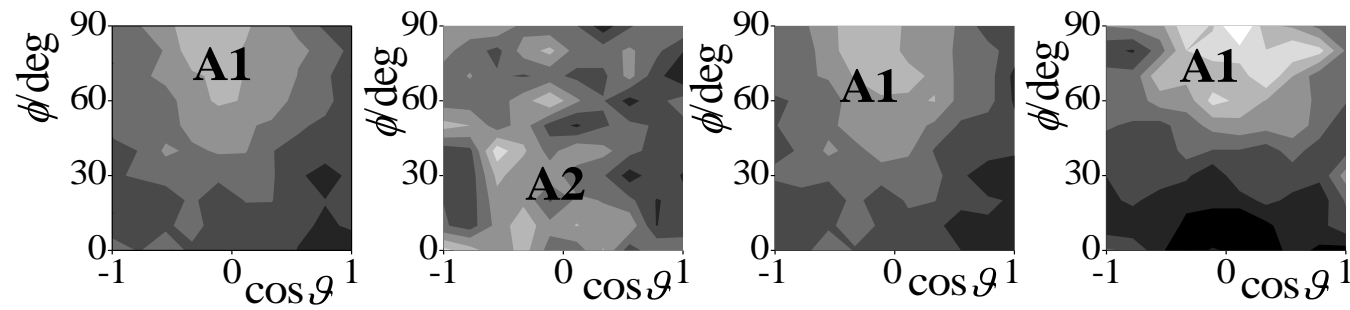

$10 \%$ acetone
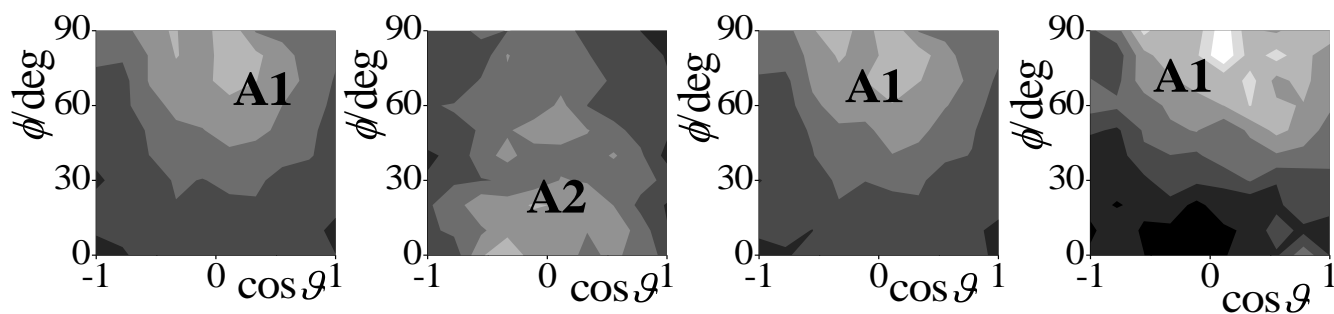

$30 \%$ acetone
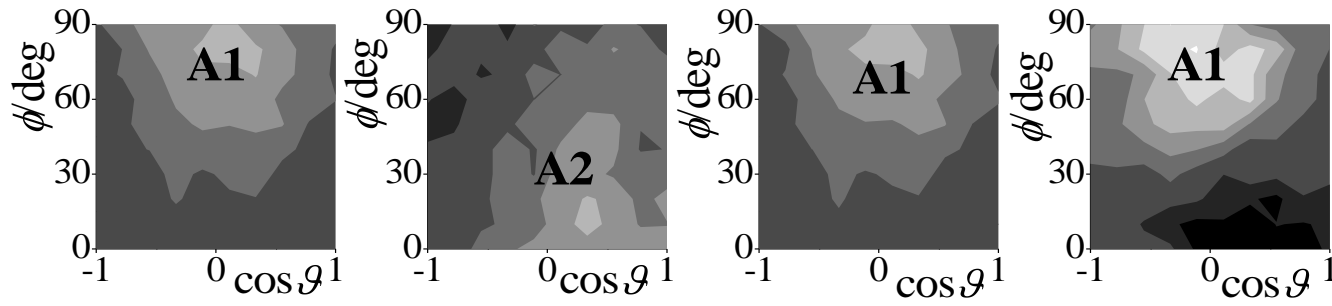

$50 \%$ acetone
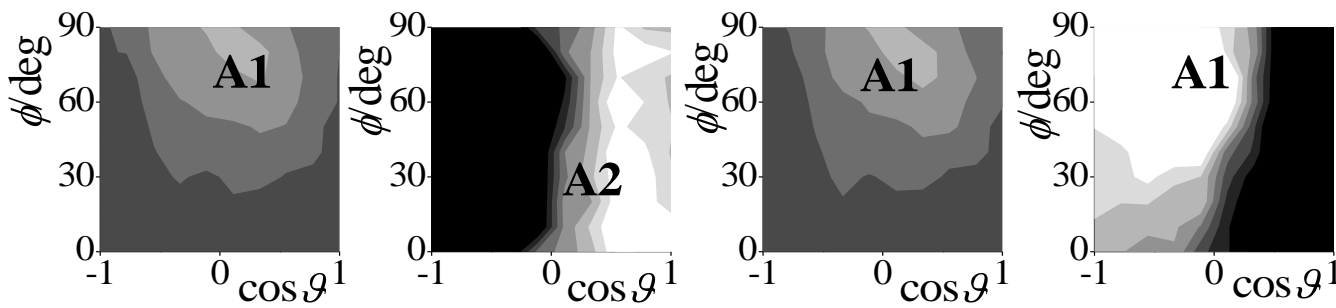

$70 \%$ acetone
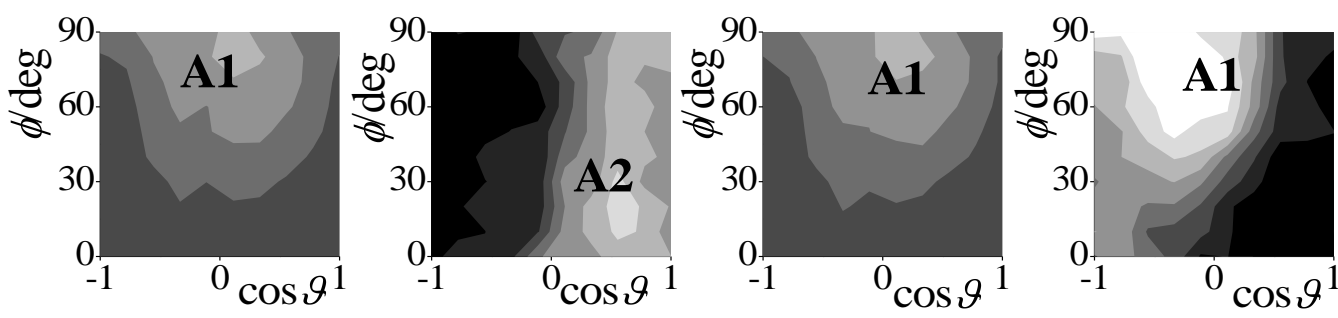

$90 \%$ acetone
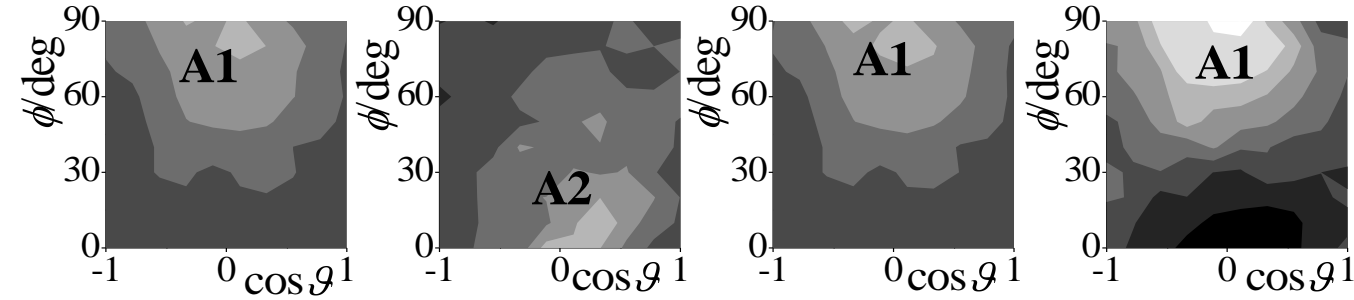

$100 \%$ acetone 
Figure 13.

Idrissi et al.

entire surface region $A$ region $B \quad$ region $C$

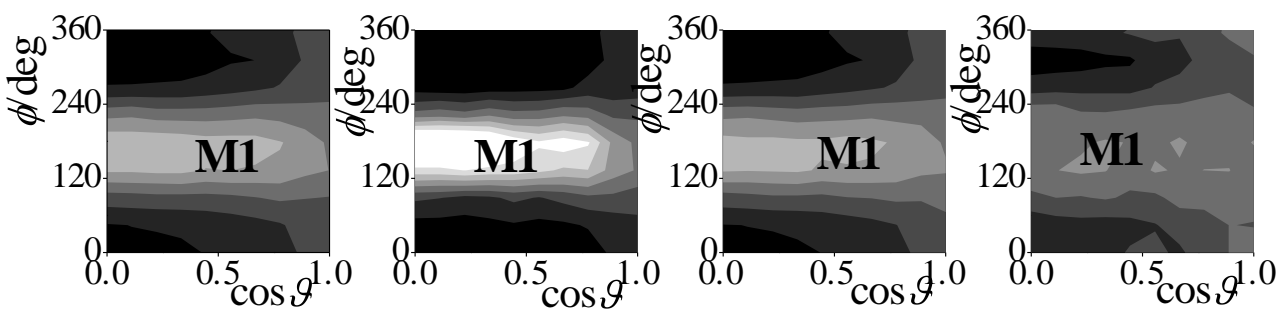

$0 \%$ acetone
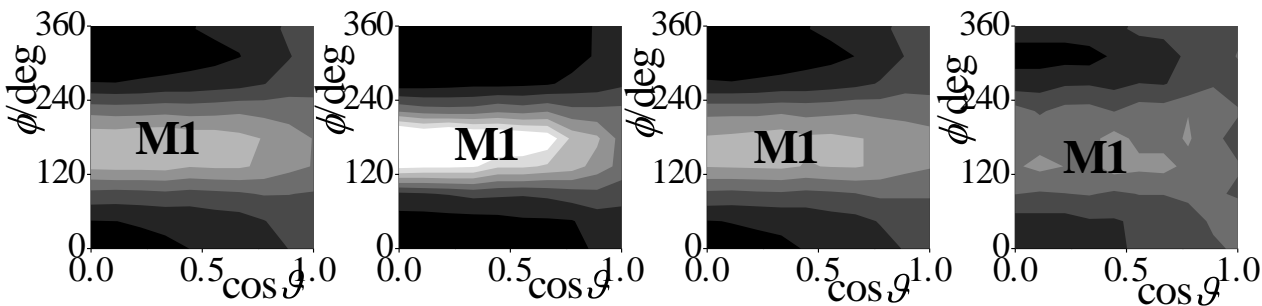

$10 \%$ acetone
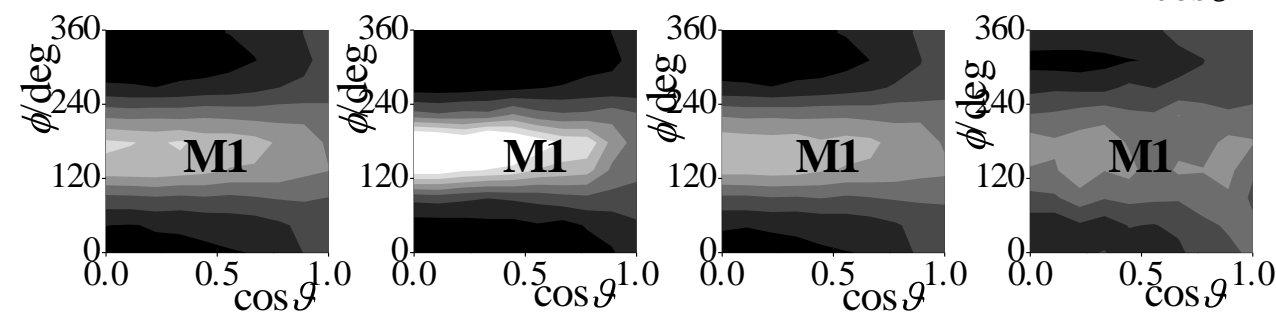

$30 \%$ acetone
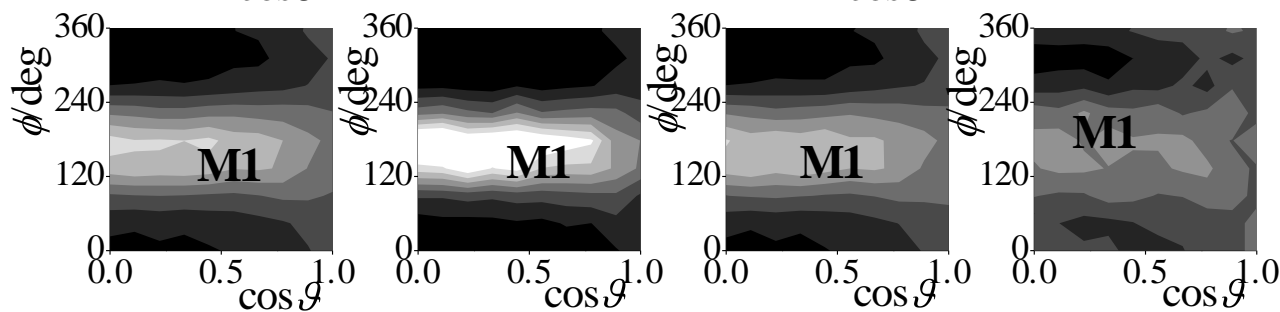

$50 \%$ acetone
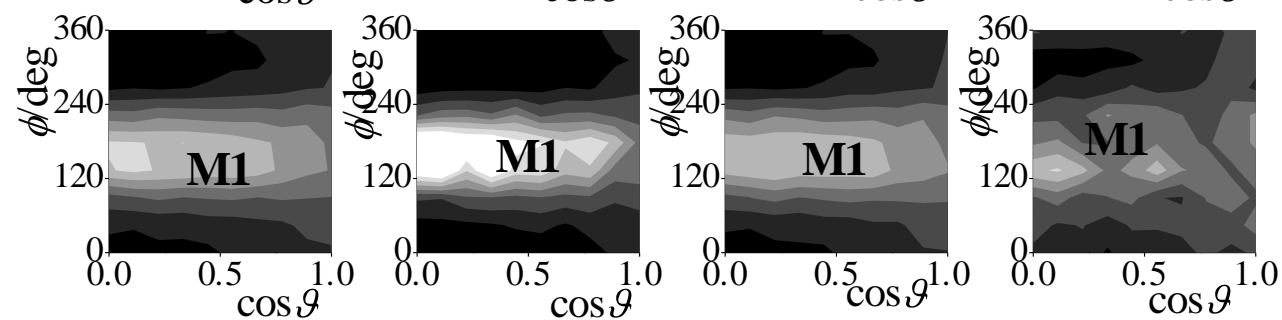

$70 \%$ acetone
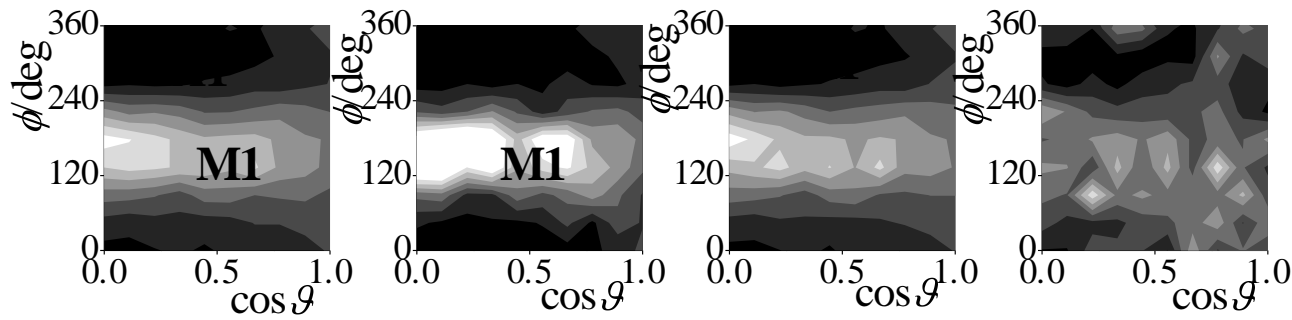

$90 \%$ acetone 
Figure 14.

Idrissi et al.
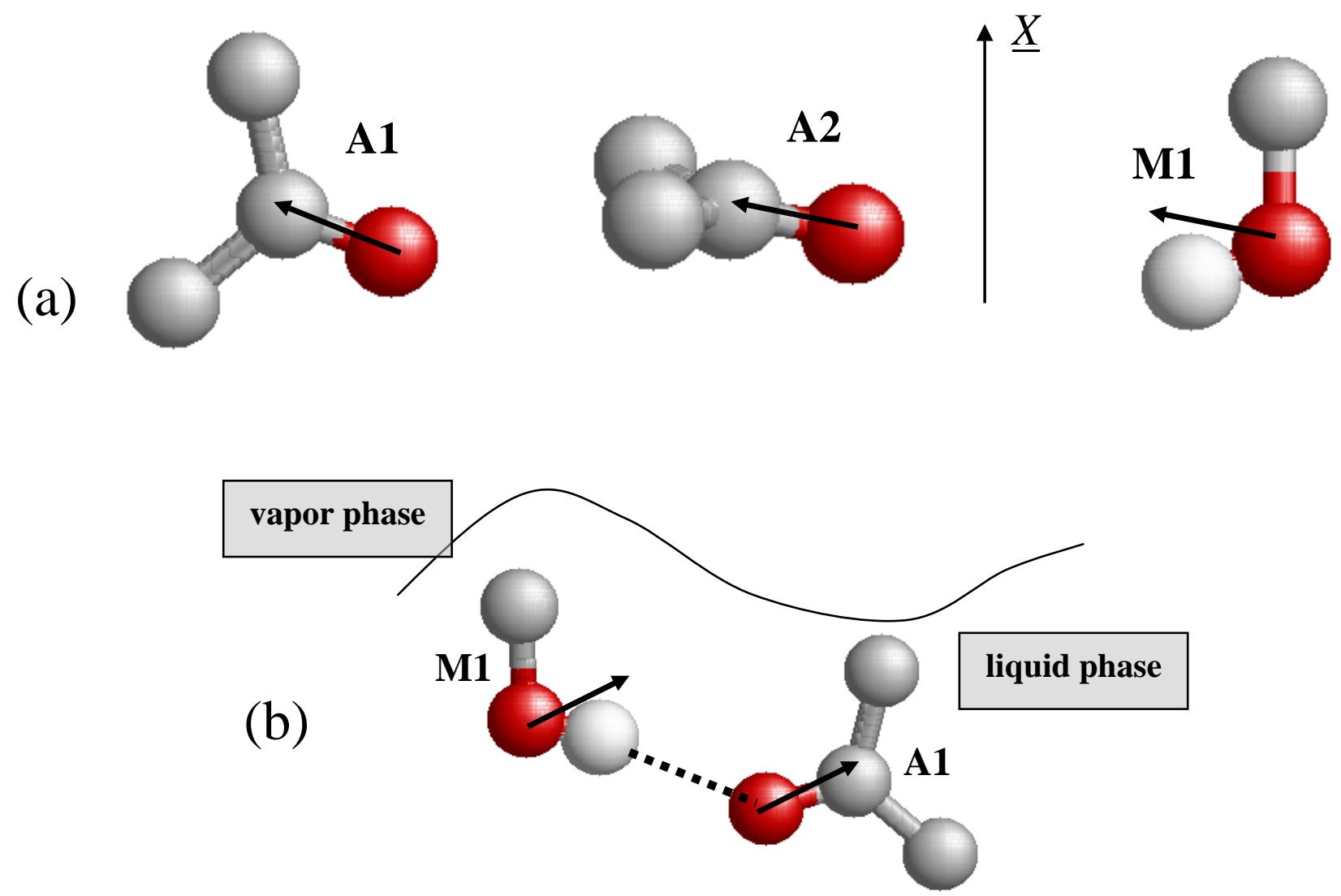